\title{
La mise en place de l'offre et la formation des usages des NTIC. Les cas de Vidéoway et de Télétel
}

\author{
Jean-Guy Lacroix, Gaëtan Tremblay et Gilles Pronovost
}

Numéro 21, 1993

L’innovation technologique

URI : https://id.erudit.org/iderudit/1002221ar

DOI : https://doi.org/10.7202/1002221ar

Aller au sommaire du numéro

\section{Éditeur(s)}

Département de sociologie - Université du Québec à Montréal

ISSN

0831-1048 (imprimé)

1923-5771 (numérique)

Découvrir la revue

Citer cet article

Lacroix, J.-G., Tremblay, G. \& Pronovost, G. (1993). La mise en place de l'offre et la formation des usages des NTIC. Les cas de Vidéoway et de Télétel. Cahiers de recherche sociologique, (21), 79-122. https://doi.org/10.7202/1002221ar
Résumé de l'article

Après avoir situé l'interaction entre la mise en place de l'offre des nouvelles technologies d'information et de communication (NTIC) et la formation des usages sociaux dans le contexte de la convergence entre

télécommunications-câblodistribution, les auteurs se donnent comme objectif d'identifier les étapes périodisant le processus d'implantation et de généralisation de la télématique, de repérer les acteurs ayant un poids structurant dans le déroulement du processus et de saisir l'action déterminante de son évolution dans chacune des périodes. Pour y parvenir, les auteurs utilisent les résultats de deux enquêtes : l'une ayant porté sur minitel; l'autre sur le cas de Vidéoway. Six périodes sont décrites et analysées : 1) l'élaboration du projet social informatique où l'État joue le rôle central; 2) l'établissement d'une norme technique et la prise en charge par des acteurs industriels de la réalisation concrète du projet; 3) la mise au point d'un prototype et sa mise à l'essai sociale qui permet d'affiner la proposition technique; 4) l'amorce de la socialisation de la nouvelle technologie par des expérimentations grandeur nature, grand public, ce qui marque l'entrée en scène des usagers en tant qu'acteurs réellement actifs dans le déroulement du processus; 5) la première phase de commercialisation, durant laquelle se poursuit la mise au point de l'offre, particulièrement au niveau des contenus, et par laquelle l'offre se donne une masse critique de consommateurs, ce qui lui permettra de légitimer ses efforts de généralisation; 6) finalement, la généralisation qui se réalise sans toutefois que l'entièreté de la population soit touchée et qui se confond avec un mouvement de diversification et de pénétration dans un ensemble élargi de pratiques sociales. En conclusion, les auteurs reviennent sur les conditions de généralisation de l'informatisation et sur le rôle de la convergence comme moment décisif du processus d'informatisation.
Ce document est protégé par la loi sur le droit d'auteur. L’utilisation des services d'Érudit (y compris la reproduction) est assujettie à sa politique d'utilisation que vous pouvez consulter en ligne.

https://apropos.erudit.org/fr/usagers/politique-dutilisation/ 


\title{
La mise en place de l'offre et la formation des usages des NTIC. Les cas de Vidéoway et de Télétel
}

\author{
Jean-Guy LACROIX, Gaëtan TREMBLAY, \\ et Gilles PRONOVOST
}

Les données et l'analyse dont ce texte ${ }^{2}$ fait part sont tirées d'un programme de recherche ${ }^{3}$ menée par deux équipes, l'une québécoise et l'autre française. Ce programme se déroule depuis trois ans et porte sur l'incidence de l'informatisation sociale sur les industries de la culture et des communications, plus particulièrement sur les industries françaises et québécoises des télécommunications et de la télédiffusion-câblodistribution. L'objectif de la recherche est de faire une analyse comparative des structures industrielles et des stratégies des entreprises, des politiques de communication, de l'organisation du travail et de la formation des usages sociaux des nouvelles technologies d'information et de communication (NTIC). Dans ce texte, nous limitons nos commentaires aux stratégies de mise en place de l'offre technologique et à la formation des usages sociaux des produits et services qui en relèvent. Plus spécifiquement, nous nous intéresserons à deux produits: Vidéoway de la firme Vidéotron et Télétel ${ }^{4}$ de France Télécom.

Notre objectif est d'identifier les étapes scandant, périodisant, le processus d'implantation et de généralisation de la télématique, de repérer les acteurs

1 Les auteurs tiennent à remercier monsieur Pierre Mœglin qui, par ses conseils et ses critiques constructives autant qu'éclairantes, a grandement facilité l'avancement de leurs travaux.

2 Ce texte a été élaboré à partir de la communication présentée par J.-G. Lacroix et G. Gagnon au dernier congrès de l'ACSALF tenu en mai 1993 à Rimouski dans le cadre du congrès annuel de l'ACFAS.

3 Ce programme a été soutenu par les ministères des Affaires internationales du Québec et des Affaires étrangères de France dans le cadre du programme de coopération internationale France-Québec. Les auteurs tiennent à les en remercier.

4 Le nom Télétel désigne une technologie qui se concrétise par le biais de différents appareils, mais de façon particulière par le minitel, le terminal. De façon générale, nous utiliserons le terme Télétel, sauf lorsqu'il sera explicitement question du terminal minitel. Notons cependant que le nom minitel a couramment été utilisé, et l'est encore, pour la commercialisation de la norme autant que pour désigner le terminal. 
structurants du processus et d'analyser leur rôle, de saisir la nature prescriptive des initiatives d'introduction et de généralisation des promoteurs et de mettre en lumière l'action déterminante de l'évolution du processus selon les différents moments de son déroulement en France et au Québec. Notre démarche comparative consiste donc à s'appuyer sur des situations homologues mais différentes afin de saisir les mécanismes sociaux structurants de l'informatisation sociale.

Pour y parvenir, nous utiliserons les résultats de deux enquêtes effectuées dans le cadre du programme de recherche décrit plus haut, enquêtes qui avaient pour but de faire l'historique du développement, de l'implantation et de la généralisation de Vidéoway et de Télétel ${ }^{5}$. Nous avons ainsi effectué deux séries d'entrevues avec des informateurs clés, c'est-à-dire des personnes ayant occupé des postes de direction ou de responsabilité dans le processus de mise en place de l'offre de ces deux technologies ${ }^{6}$. Nous avons également pris en compte la documentation pertinente recueillie auprès des interviewés et des entreprises promotrices.

Les pages qui suivent exposent succinctement les principaux éléments de notre problématique, résument les principaux résultats de nos enquêtes et, en conclusion, rassemblent de façon synthétique nos remarques principales concernant la périodisation de la mise en place de l'offre et de la formation des usages sociaux sous l'impact de la démarche prescriptive des promoteurs?

5 G. Gagnon, J.-G. Lacroix et G. Tremblay, Historique de Vidéoway, Montréal, GRICIS, 1992, 39 pages; G. Gagnon, J.-G. Lacroix et P. Pajon, Usages du minitel. La stratégie de formation des usages du minitel: 1983-1984, Montréal, GRICIS, 1993, 84 pages.

6 Au total, neuf entrevues: quatre au Québec chez Vidéotron; cinq en France, dont quatre chez France Télécom et une auprès d'un chercheur CNRS ayant participé à l'expérience de Vélizy.

7 Dans ce texte, nous n'abordons que les initiatives de développement, d'implantation et de généralisation. L'aspect discours de la démarche prescriptive est délaissée pour des raisons évidentes d'espace. Nous avons toutefois touché cet aspect dans notre programme de recherche. Voir P. Rigaud et J.-G. Lacroix, VidéotronVidéoway. Discours promotionnels et prescriptions d'usages, Grenoble, GRESEC, 1992, 117 pages. Également, L. Santerre, P. Mœglin et J.-G. Lacroix, Le discours prescriptif de France Télécom et la production des usages sociaux de la télématique, rapport de recherche, Paris-Grenoble, GRUSEM-GRESEC, 1993, en rédaction. Cette étude montre que, dans le cas de Télétel, le discours prescriptif de France Télécom s'inscrit dans trois grands types de stratégie discursive: promotionnelle, politicoprospective et spécifiquement prescriptive (pour encadrer et guider les pratiques à l'aide de modes d'emploi). 


\section{La convergence: un enjeu sociétal ${ }^{8}$}

Dans les années 70, le téléviseur s'est vu attribuer par les futurologues le potentiel de terminal multi-usages autour duquel devaient s'intégrer les divers moyens de communication. Puis, les jeux vidéo, la micro-informatique et le vidéotex se sont développés de façon relativement indépendante, et on a moins parlé du téléviseur comme centrale de communication. Plus récemment, le discours sur la convergence technologique a connu un regain de vie avec la miniaturisation de l'informatique, la baisse des coûts d'accès aux produits et services informatiques et, surtout, télématiques, la croissance de la capacité de stockage de l'information informatisée, la généralisation de la numérisation des signaux, les progrès de la fibre optique et des réseaux de micro-ondes et la création des réseaux à large bande. De nouveau, et plus que jamais, on rêve de l'intégration des télécommunications, de l'informatique et de la télédiffusion-câblodistribution. Tout récemment, le projet d'autoroute électronique (Information Highway) du président Clinton ${ }^{9}$, les projets américains de DDS (diffusion directe par satellite) ${ }^{10}$ que les câblodistributeurs canadiens ont qualifiés d' "étoiles de la mort" lors des audiences du CRTC tenues en mars $1993^{11}$ et leur projet d'adressabilité universelle qui y répond et qu'a accepté le CRTC $^{12}$ indiquent clairement que cette perspective est plus que du rêve, qu'elle représente un possible nettement probable et que certains acteurs sont convaincus de pouvoir en tirer profit et travaillent à le concrétiser.

La perspective de la convergence, comme on le voit, s'appuie sur le progrès technologique. Mais, même si l'informatique est de plus en plus utilisée dans le secteur de l'audiovisuel, et même si la télédiffusion utilise depuis longtemps les systèmes de télécommunication, l'intégration des trois technologies n'en est encore qu'à ses débuts. En effet, rien n'est encore joué parce que le processus d'informatisation sociale ne dépend pas uniquement de la technologie, mais aussi

8 Les principaux éléments de cette problématique sont tirés du texte définissant la problématique générale du programme de recherche sommairement décrit dans l'introduction de ce texte. Voir J.-G. Lacroix, B. Miège, P. Mœglin, P. Pajon et G. Tremblay, "La convergence des télécommunications et de l'audiovisuel. Un renouvellement de perspective s'impose", Technologies de l'information et société (TIS), vol. 5, no 1, 1993, p. 81-105; également publié dans Telos, "La convergencia entre telecomunicationes y audiovisual. Por una renovacion de perspectivas", no 34 , p. 89-103.

9 Voir à ce sujet, P. Elmer-Dewitt, "Electronic Superhighway", Time, 12 avril, 1993, p. 48-53; également, A. Hills Moore et A. J. Michels, "The Race to Rewire America", Fortune, 12 avril, 1993, p. 42 et suiv.

10 G. Wall, R. Poirier et A. Boucher, Le rapport de la DDS, Ottawa, ACTC, 1992.

11 Voir à ce propos J.-G. Lacroix, G. Tremblay, M. Saint-Laurent et M. Ménard, “'Étoiles de la mort' ou épouvantails?", Le Devoir, 19 avril, 1993, p. A-7; des mêmes auteurs, "Câblodistribution 2001: ce que le public risque de devoir payer!", La Presse, 23 avril 1993 , p. B-3.

12 G. Tremblay, J.-G. Lacroix, M. Ménard et M. Saint-Laurent, "Les abonnés du câble devront dépenser en moyenne deux fois plus”, La Presse, 18-06-1993, p. B-3. 
des politiques de communication et de développement industriel des États, des structures industrielles en place et des stratégies des entreprises qui y œuvrent et des usages sociaux des technologies en place et de ceux qui sont en voie de formation. Au Québec, le système Vidéoway, dont la commercialisation a commencé en 1990, constitue un point de cristallisation des aspirations à la convergence et un élément potentiellement moteur de la généralisation de l'informatisation. En France, le système Télétel, dans les années 1980, et, plus récemment, les applications du réseau Numéris ont joué le même rôle ${ }^{13}$.

\subsection{L'informatisation sociale et l'État}

Les politiques et les interventions étatiques ont joué, et continuent d'avoir, un rôle fondamental dans le processus d'informatisation sociale. En tant qu'appareil centralisé de gestion et de maintien de l'intégration sociale, du consensus et de régulation économique et de protection des intérêts nationaux (encore, malgré la mondialisation), l'État condense, sous l'influence des différents groupes sociaux et de certains groupes de pression, les problématiques sociales. Et il se doit de répondre à la demande sociale ${ }^{14}$ qui émane du rapport social, particulièrement des structures et des logiques industrielles lorsqu'elles sont en crise.

Témoignent de ce rôle les grandes commissions d'enquête mises sur pied par les États occidentaux au cours des années soixante-dix et quatre-vingt pour étudier la question de l'informatisation de la société et leurs discours politico-prospectifs qui visaient à préparer les esprits à accepter la réorganisation socioéconomique autour des technologies informatiques ${ }^{15}$. Les initiatives étatiques qui suivirent la

13 On doit retenir, toutefois, qu'en France l' "aspiration" à la convergence a été fortement limitée par la faiblesse des réseaux câblés, par l'action de l'État qui a voulu favoriser la survie de la section micro-informatique de Thomson et par le fait que le développement de la filière image-câblée n'a été principalement le fait que de Canal Plus.

14 Par demande sociale, nous entendons les problématiques sur lesquelles la société se penche en tant que collectivité structurée. Ces questions émergent dans le champ politique parce qu'elles naissent de la dialectique des rapports sociaux et, ainsi, exigent une réponse collective qui souvent passe par une renégociation du contrat social ou du moins d'une partie de celui-ci. La demande sociale résulte donc de l'interrelation entre les conditions infra-structurelles de l'action sociale, "l'opinion" du grand public, les volontés et les points de vue des décideurs dans les appareils productif et administratif, ou du moins des "fonctionnaires" de ceux-ci, et les réponses et interpellations que travailleurs, grand public, entrepreneurs et classe politique s'adressent dans le cadre des rapports sociaux.

15 À titre d'exemple mentionnons, pour la France: les rapports Nora-Minc, L'informatisation de la société, Paris, La Documentation française, 1978; et, Glowinski, Télécommunications. Objectif 2000, Paris, Dunod, 1980; pour le Canada et le Québec, les rapports: Un univers sans distance, Ottawa, Information Canada, 1971; L'arbre de vie, Ottawa, MCC, 1972; Dimension d'une politique de télé-informatique 
tenue de ces commissions et la parution des rapports qui en émanèrent visaient à développer la technologie, puis à soutenir la prise en charge du projet d'informatisation sociale par des acteurs industriels, pour finalement favoriser la mise en place d'une offre concrète "prétendant" être "la" traduction de ce projet.

\subsection{Les structures industrielles et les stratégies de mise en place de l'offre des NTIC}

Les structures industrielles sont lentes à se mettre en place, mais une fois esquissées, elles tendent à se stabiliser et à se reproduire. Elles constituent ainsi des contraintes qui conditionnent l'action ou même "déterminent" les stratégies des entreprises actives dans une industrie donnée autant que dans les industries connexes ou complémentaires. Cependant, à certains moments de leur histoire (à l'occasion de crise, sous l'impact de certaines innovations ou d'énoncés politiques, etc.), des industries sont transformées par l'action de certains acteurs, qui toutefois, il faut le rappeler, ont été modelés par les structures dans lesquelles et par lesquelles ils se sont développés. Dans certains cas, ces innovateurs appartiennent à l'industrie elle-même. Dans d'autres cas, ils proviennent d'autres industries.

Les stratégies d'acteurs peuvent donc avoir un effet de stabilisation sur la structure et la logique d'une industrie ${ }^{16}$ ou au contraire un effet de transformation, même de restructuration. Cependant, les stratégies qui pourraient mener à des transformations structurelles ne sont pas le fait de n'importe quel acteur. En effet, ce potentiel de restructuration dépend du poids relatif des acteurs (par exemple, leur taille et leur position dans le marché), de leur capacité économique (leurs performances économiques, leur place stratégique ou non dans une conjoncture de réorganisation, etc.), et de leur volonté de restructuration et d'innovation, ce qui dépend à la fois de leur intérêt pour la technologie informatique, la convergence et l'intégration et de leur vision du devenir de l'industrie à laquelle ils appartiennent et de celui des industries concurrentes ou connexes. Tout cela se traduit quelquefois par un certain volontarisme industriel ${ }^{17}$ privé (marchand) ou étatique. C'est manifestement le cas de Vidéotron et de France Télécom ${ }^{18}$.

pour le Québec, Québec, MCQ, 1976; Bâtir l'avenir, Québec, MCQ, 1982; et Le Québec et les communications: un futur simple?, Québec, MCQ, 1983.

16 Au sujet des logiques dans le domaine des médias, voir B. Miège, P. Pajon et J.-M. Salaün, L'industrialisation de l'audiovisuel. Des programmes pour les nouveaux médias, Paris, Aubier, 1986; également, G. Tremblay et J.-G. Lacroix, Télévision. Deuxième dynastie, Québec, Presses de l'Université du Québec, 1991.

17 P. Mœglin, "Télématique: De la recherche sur les usages aux usages de la recherche", Bulletin du CERTEIC, no 12, 1991, p. 23-50.

$18 \mathrm{Ce}$ volontarisme ne se traduit pas toujours de façon marquante ni facilement en convergence intersectorielle. Ainsi, Vidéotron a mis un certain temps avant de manifester des prétentions du côté des télécommunications entre autres par la mise sur pied de sa filiale Vidéotron Communications Ltd et, plus récemment, par une entente 
1.3 La formation des usages sociaux des NTIC et les différents moments du processus d'informatisation sociale

Dans le processus d'implantation et de généralisation des NTIC, c'est donc l'offre qui joue le rôle initiateur et par la suite moteur, y compris au niveau du développement des usages sociaux, ce qui ne veut pas pour autant dire que les usagers n'ont aucun poids dans le processus. En effet, ce sont les usages sociaux en place et ceux en formation qui favorisent ou empêchent l'implantation et la généralisation des innovations techniques, la domination d'une innovation plutôt qu'une autre, et, donc, la domination d'une entreprise, d'une filière technoéconomique ou d'une logique industrielle plutôt que d'autres. S'il y a antériorité de l'offre, c'est la réponse du public qui sanctionne l'innovation et les stratégies d'implantation et de généralisation des produits et services concrétisant l'informatisation sociale ${ }^{19}$. Ainsi, le processus d'implantation et de généralisation des usages des NTIC relève de l'interaction entre la mise en place de l'offre technologique et la structuration de la demande par la formation des usages sociaux, ce qui ne se fait pas du jour au lendemain.

La liste des transformations du minite ${ }^{20}$ et celles des péripéties de la mise au point du système Vidéoway montrent que les NTIC ne se stabilisent que lentement et qu'elles continuent à être modifiées au fur et à mesure de leur implantation et même au cours des phases subséquentes de leur généralisation. Nous avons donc affaire à des utilisations et non à de véritables usages sociaux ${ }^{21}$ tant et aussi longtemps que la technologie nouvelle et les produits et services qui la concrétisent ne sont pas stabilisés et ne font pas l'objet d'utilisations récurrentes organiquement insérées dans la quotidienneté de la généralité des citoyens. Autrement dit, tant et aussi longtemps qu'elles n'ont pas été généralisées, donc qu'elles ne font pas intégralement partie du processus de socialisation des individus dans une société donnée et que la production-consommation (les utilisations) des produits et services qui en découlent ne s'est pas cristallisée en norme sociale. Cela implique d'une part que le processus de formation des usages sociaux est aussi

commerciale avec Unitel (le concurrent de Bell dans le secteur des télécommunications). De son côté, France Télécom, sortant de son métier de base, les télécommunications, a connu, et connaît encore, de nombreux déboires sur les réseaux câblés en fibre optique. Cela n'a toutefois pas empêché l'entreprise d'investir récemment, à titre d'opérateur de réseaux, dans la câblodistribution française.

$19 \mathrm{Au}$ sujet de l'incidence structurante de la dynamique sociale sur les moyens de communication, voir P. Flichy, Une histoire de la communication moderne, espace public et vie privée, Paris, La Découverte, 1991.

20 P. Mœglin, "Télématique: De la recherche sur les usages aux usages de la recherche", article cité, p. 39.

21 Par usages sociaux, nous entendons des modes d'utilisation se manifestant avec suffisamment de récurrence, sous la forme d'habitudes suffisamment intégrées dans la quotidienneté, pour s'insérer et s'imposer dans l'éventail des pratiques culturelles préexistantes, se reproduire et éventuellement résister en tant que pratiques spécifiques à d'autres pratiques concurrentes ou connexes. 
modelé par le contrôle social et qu'il renferme des aspects de contrainte, d'imposition, autant que de stimulation, de séduction, et, d'autre part, que cette dynamique contradictoire (la séduction-contrainte ${ }^{22}$ ) est présente tout au long du processus. Cependant, la force des contraintes et de la séduction comme les formes et les modalités par lesquelles elles s'expriment diffèrent selon le moment du processus de formation des usages sociaux. Ce dernier se concrétise ainsi à travers l'interaction de la démarche prescriptive de l'offre (par les promoteurs politiques autant qu'industriels et commerciaux) et des réactions des consommateurs (de tout ordre: institutions, entreprises, associations ou individus et familles).

Présente tout au long du processus, mais sous des formes et des modalités différentes selon les étapes, la démarche prescriptive se fait, d'une part, par le biais d'actions, d'initiatives, de développement de la technologie et de tactiques d'introduction et de généralisation de celle-ci, ce qui inclut "l'imposition" d'innovation à des ensembles plus ou moins grands de consommateurs, comme nous le verrons plus loin; et, d'autre part, par le biais d'appareils et de services, et par le biais du discours prescriptif et promotionnel de toutes formes (ce qui inclut les déclarations publiques) qui accompagne, structure et organise la conversion de l'innovation technique en média et les utilisations en usages sociaux. Le rôle de ce discours est donc de préparer les marchés en stimulant les consommateurs et en formant les utilisateurs et d'atténuer autant que possible, à chaque étape ${ }^{23}$, le décalage et la tension que l'antériorité de l'offre tend inévitablement à induire entre les utilisations prescrites et les utilisations effectives des usagers.

Toutefois, le processus de formation des usages sociaux est d'autant plus complexe que l'informatisation sociale se réalise à travers une diversité de filières techno-économiques (particulièrement l'informatique, les télécommunications et la télédistribution) et de produits et services (grands ordinateurs centraux, microordinateurs, ordinateurs portatifs, télématique, petits appareils de jeux, guichets bancaires automatiques, bornes interactives, agendas électroniques, bloc-notes

22 R.J. Ravault emploie la notion de "coerséduction" pour décrire le fonctionnement contradictoire de certains mécanismes, réseaux, de socialisation ou d'acculturation. Voir, "Défense de l'identité culturelle par les réseaux traditionnels de 'coerséduction", International Political Science Review, Sage (USA), 1986, vol. 7, no 3, p. 251-280; et, "Resisting Media Imperialism by Coerseduction", Intermedia, Londres, 1985, vol. 25 , no 3 , p. 32-37. Pour notre part, l'emploi simultané des termes coercition ou contrainte et séduction a une ambition plus modeste et plus spécifique. Nous ne voulons ainsi que qualifier des aspects du processus de prescription des usages, donc que certains éléments de la stratégie des offreurs-promoteurs de NTIC.

23 J.-G. Lacroix, P. Mœglin et G. Tremblay, "Usages de la notion d'usages: NTIC et discours promotionnels au Québec et en France", Les nouveaux espaces de l'information et de la communication, Actes du huitième congrès national des sciences de l'information et de la communication, Lille, 1992 p. 239-248. À propos de la temporalité dans le développement et l'implantation des NTIC voir également P. Flichy, "Cornmunication: progrès technique et développement des usages", Rapport à la CEE, Réseaux, no 24, mai 1987, p. 105-110. 
informatisés, par exemple le Newton de Macintosh, "scanners"...). L'informatisation de la société ne peut résulter que de l'articulation de cette diversité en un ensemble d'objets techno-économiques relativement stable, en système ${ }^{24}$, ce qui est loin d'être encore le cas même si des signes certains de convergences se manifestent, particulièrement en Amérique du Nord. Aussi, pour le moment, l'interrogation scientifique sur le processus d'informatisation sociale se doit de se situer au croisement des initiatives d'introduction et de généralisation des NTIC ayant le plus d'incidence sur l'articulation en système cohérent et intégrateur de la diversité des NTIC et sur le procès de socialisation des usagers, tout en tenant compte du fait que les utilisations n'ont pas encore le caractère stable et récurrent des véritables usages sociaux et qu'elles n'ont par encore le caractère d'une familiarisation transversale à la diversité des produits et services qui actuellement concrétisent le procès et le projet d'informatisation sociale. C'est la raison pour laquelle nous avons centré notre programme de recherche sur la filière télématique en faisant l'hypothèse que la perspective de la convergence, repérable dans certains discours et certaines initiatives, marque un moment crucial, un moment de basculement du processus vers la généralisation.

\section{La mise en place de l'offre de Vidéoway et de Télétel}

\subsection{Le politique et le projet social informatique (période 1)}

Nous avons déjà souligné que le processus d'informatisation sociale commence par l'élaboration d'un projet de société informatisée et d'un certain discours politico-prospectif de la part des États. Dans ce texte, nous n'analyserons pas cette première période du processus d'informatisation afin de mieux nous concentrer sur les étapes de mise en place plus concrètes de l'offre, c'est-à-dire à partir du moment où on tente de développer la technologie comme telle, de l'implanter et de la généraliser ${ }^{25}$.

Cependant, pour bien situer le contexte socio-historique dans lequel s'enclenche le processus, il est utile de rappeler que le projet d'informatisation de la société et le discours politico-prospectif qui le cristallise dans l'espace public "voient le jour" au moment même où l'on parle de crise structurelle, au milieu des années 1970, et où la dialectique sociale est "demandeuse" d'une solution unifiée et faisant consensus dans la collectivité. C'est parce que la structuration dans la dynamique sociale dépend du déjà structuré et parce que l'État est devenu, sous le

24 G. Tremblay et M. Sénécal, "La science des communications et le phénomène technique", Sciences sociales et transformations technologiques, actes d'un colloque, Québec, Conseil de la science et de la technologie, doc. 87.02, 1987.

25 Dans notre programme de recherche, nous nous sommes toutefois penchés sur cette question. Voir M. Metzener, G. Tremblay et J.-G. Lacroix, Analyse des discours politico-prospectifs sur les nouvelles technologies d'information, Montréal, GRICIS, septembre 1992, 135 pages. 
fordisme, interventionniste et qu'il joue un rôle central et direct dans la régulation sociale, que, d'une part, la réponse au diagnostic d'entrée en crise structurelle commence par un discours qui a comme objectif de préparer les esprits ${ }^{26}$, de convaincre toute la collectivité de la nécessité et de l'“inévitabilité" de la solution informatique et, d'autre part, que l'acteur central de cette amorce de réponse sociale est l'État. Aussi, autant au niveau de l'établissement du diagnostic, que de la mise en train de l'opération de recherche de la solution, que de celle de légitimation de celle-ci, l'État s'impose comme l'acteur premier, principal et organisateur de la mise en place des conditions politiques, économiques et sociales de réponse à la crise.

Toutefois, et en cela on retrouve l'incidence du déjà structuré, ces conditions de réponse, comme la forme concrète du diagnostic et des initiatives avancées en réponse, varient d'un champ socioéconomique national à l'autre. Ainsi, le centralisme beaucoup plus grand de l'État français et son interventionnisme beaucoup plus général et systématique que ceux de l'État canadien expliquent non seulement la forme achevée et directive du projet d'informatisation de la société française, mais aussi la trajectoire beaucoup plus rectiligne du processus de mise en place de l'offre technologique, comme l'ampleur des ressources qui y furent consacrées, tout autant que le rythme beaucoup plus rapide de réalisation (passage du projet à l'implantation, puis à la généralisation).

\subsection{La mise au point de la technologie et les premières expériences techniques} (période 2)

Les premières recherches canadiennes sur le vidéotex ont été l'œuvre du ministère des Communications (MCC) ${ }^{27}$. On élabora tout d'abord une réplique du système anglais Prestel. Puis, on mit au point, en 1979, un système de vidéotex dont l'originalité résidait dans ses "instructions de description de l'image 28 ". Ce sera le système Télidon, qui toutefois s'avérera très coûteux. En effet, le terminal Mark I, fabriqué par la compagnie Norpak d'Ottawa, coûtait environ 2600 \$. Il n'est donc pas étonnant qu'il fut surnommé la Cadillac des vidéotex ${ }^{29}$.

26 B. Miège et Y. de la Haye, "De l'ère de la communication aux marchés de la communication", Communication Information, vol. 6, nos $2 / 3$, hiver 1984 , p. 209 212.

27 J.-C. Guédon, "Norme ou système technique? Les avatars de Télidon au Canada", Réseaux-TIS, no 37 et vol. 2, no 1, 1989, p. 69-82 et p. 69-80.

28 Code sténographique basé sur des formes géométriques (le point, la ligne, l'arc, le polygone et le rectangle) alors que Prestel (anglais) et Télétel (français) n'avaient comme base que le carré.

29 P. Desbarats, Les quotidiens et l'ordinateur, une industrie en transition, Ottawa, Commission royale sur les quotidiens, 1981, p. 55. 
Voulant promouvoir "son" système de vidéotex, l'État canadien stimula l'expérimentation sur la technologie Télidon et soutint financièrement les efforts de développement de plusieurs entreprises. En 1981, cinq entreprises de téléphone ${ }^{30}$, deux entreprises de radiodiffusion ${ }^{31}$ et une entreprise de câblodistribution, Télécâble Vidéotron, menaient des expériences sur des systèmes de vidéotex ou de télétexte à partir de la technologie Télidon. Deux de ces expériences furent menées au Québec: IRIS à Radio-Canada et SID-Télidon ${ }^{32}$ chez Vidéotron.

Le MCC s'était, dès $1979^{33}$, associé à Vidéotron en investissant 1,85 millions de dollars dans le développement de SID-Télidon ${ }^{34}$. À ce moment, Vidéotron commençait à s'intéresser à d'autres possibilités de transmission que la câblodistribution conventionnelle ${ }^{35}$. Plus particulièrement, on voulait offrir de nouveaux services aux abonnés tout en leur permettant de n'utiliser que les services désirés ${ }^{36}$. Toutefois, derrière cet objectif se cachait la volonté des propriétaires de systèmes de télédistribution d'enrayer le piratage de la télévision payante qui privait alors les câblodistributeurs et les canaux spécialisés, tous distribués par le câble, d'une part relativement importante de revenus.

Cependant, en 1983-1984, après avoir investi 40 millions de dollars dans l'aventure du vidéotex, le MCC met fin à son programme d'aide aux entreprises privées, leur laissant ainsi le soin et la responsabilité des développements futurs ${ }^{37}$.

30 British Columbia Telephone Cie, Alberta Government Telephone, Manitoba Telephone System, Bell Canada, New Brunswick Telephone Cie.

31 Ontario Educational Commission Authority et Radio-Canada.

32 Le projet SID fut l'ancêtre du Vidéoway. Il s'agissait d'un projet de recherche visant à développer un système d'information à domicile accessible par le câble.

33 Bell Canada a également expérimenté - dans le cadre du projet Vista, produit d'une entente, 1979, de dix millions de dollars entre le MCC et Bell (J.-C. Guédon, "Norme ou système technique? Les avatars de Télidon au Canada", article cité, p. 74) - auprès d'un petit groupe d'utilisateurs à Cap Rouge en banlieue de Québec la technologie Télidon. Cependant, la majeure partie des expérimentations sur Vista ont été faites dans la région de Toronto. Dans ce texte, nous ne pouvons tenir compte de ces expériences étant donné les limites d'espace. Nous les avons toutefois pris en considération dans notre programme de recherche. Il en a été de même pour Antiope dans le cas français.

34 Voir à ce sujet, Vidéotron, Présentation au Conseil de la radiodiffusion et des télécommunications canadiennes, 15 mai 1980, p. 6, cité par J.-G. Lacroix et R. Pilon, "Vidéotron: l'empire de la câblodistribution au Québec", Négocier le virage technologique, Québec, Montréal, CEQ-ICEA, 1983, p.157-175.

35 Le transport par câble d'émissions offertes par différents programmateurs-diffuseurs.

36 Les services que Vidéotron proposait alors étaient la gestion de l'énergie, la distribution de logiciels, un télétexte à haute capacité, un vidéotex (Télidon), un service de surveillance, l'accès à des banques de données et un service de télégestion. Groupe Vidéotron, Info SID Telidon II, Bulletin d'information, vol. 1, no 1, décembre 1980, p. 1 .

37 P. Desbarats, Les quotidiens et l'ordinateur, une industrie en transition, op. cit., p. 56. 
Très peu d'entre elles étaient prêtes et disposées à assumer ce rôle. Seuls Bell Canada et Vidéotron répondirent vraiment à "l'appel". La première toutefois avec comme seules motivations de préserver sa position dans sa propre industrie, la téléphonie, devant d'éventuels concurrents, dont le Télétel ${ }^{38}$, et de se réserver une place dans l'hypothétique marché de la télématique. La seconde, par contre, avec un projet relativement précis qui constituait un des axes de sa stratégie d'ensemble ${ }^{39}$. C'est seulement cet acteur, Vidéotron, qui alors manifesta une véritable volonté de concrétiser le projet de vidéotex.

Ces événements montrent que la seconde période du processus d'informatisation sociale au Canada obéit aux tendances structurelles de l'État canadien. Elle est en effet marquée par la fragmentation régionale, la canadianisation et aussi par la privatisation qui historiquement ont structuré les interventions de cet État dans le secteur des médias ${ }^{40}$. Aussi, le passage du projet d'informatisation sociale à la mise en place d'une proposition technologique concrète se fait avec un changement d'acteur au poste de commande et à travers une coupure dans la trajectoire de réalisation. Le processus souffrira de la baisse d'intérêt due à l'impératif de la rentabilité à court terme dominant la sphère privée, ce qui aura une incidence directe sur le rythme de mise au point d'une proposition techno-économique concrète et de son implantation sociale. Cela explique aussi que le projet ne pouvait être repris que par un acteur dont la position objective nourrissait un intérêt subjectif suffisant pour qu'il se fasse volontariste.

Cette seconde période se déroule en France dans des conditions infrastructurelles très différentes. Nous avons déjà souligné que l'État français est beaucoup plus centralisé, interventionniste et directif, entre autres dans les secteurs des médias, des télécommunications et de la culture en général. Les acteurs, et donc les stratégies, dans les secteurs concernés par le projet d'informatisation sociale ont ainsi dès le départ été connectés sur "le" politique, ce qui a imprimé une trajectoire plus directe et un rythme de mise en place de l'offre concrète beaucoup plus rapide.

Pour contrer l'envahissement informatique américain et trouver un débouché aux structures et équipements mis en place dans le but de rattraper le retard pris dans le domaine des télécommunications, la France fit de l'implantation du vidéotex interactif un projet de société qui, par ailleurs, devait aussi permettre à l'industrie électronique française de se tailler une place sur les marchés national et international des nouvelles technologies. Mais, avant que ce projet s'inscrive dans l'espace public grâce au rapport Nora-Minc, en 1978, et qu'il connaisse un début

38 G. Tremblay et J.-G. Lacroix, Télévision. Deuxième dynastie, op. cit., p. 113-114. 39 Ibid., p. $112-115$ et $128-130$.

40 J.-G. Lacroix, "Le rôle de la télématique dans la question du Québec", Les cahiers $d u$ socialisme, no 14, printemps 1984, p. 191-222: également, J.-G. Lacroix et R. Pilon, Câblodistribution et télématique grand public: historique du développement de la câblodistribution au Canada (1950-1980), Montréal, GRICIS, 1983, p. 2-3. 
de réalisation - par le biais de deux projets expérimentaux, Télétel $3 \mathrm{~V}$ à Vélizy et l'annuaire électronique en Ille-et-Vilaine - certains travaux de développement technologique avaient déjà ouvert la voie française de l'informatisation.

En effet, avant ces deux expériences, deux centres de recherche ont travaillé à l'élaboration d'une norme technique française. Il s'agit du CNET (Centre national d'études des télécommunications), un organisme indépendant en relation avec la DGT, et du CCETT (Centre commun d'études de télécommunications et de télédiffusion), un laboratoire partagé par la DGT et TDF ORTF). Les efforts du CNET portèrent sur la mise au point d'un système alliant informatique et téléphone. Ce système sera nommé "vidéotex interactif". De son côté, le CCETT élabora un système basé sur l'utilisation de l'intervalle de trame pour afficher des pages de texte sur un écran de télévision. Cette technique porte aujourd'hui le nom de "télétexte". En 1976, les deux centres de recherche ont uni leurs efforts pour qu'une norme commune de visualisation soit adoptée, ce sera la norme de type alphamosaïque $^{41}$ Antiope $^{42}$. Ce nom fut également employé pour désigner la version commerciale du système de télétexte principalement utilisé dans le soustitrage pour malentendants. Le vidéotex interactif reçut quant à lui le nom de Télétel ${ }^{43}$.

En 1978, la DGT (Direction générale des télécommunications des PTT, aujourd'hui France Télécom) prend la décision d'avancer dans le domaine télématique à grande diffusion en lançant deux opérations parallèles: d'une part Vélizy, qui aura comme objectif de construire une offre multi-fournisseurs de service pour grand public; et, d'autre part, l'annuaire électronique et le développement d'un terminal particulier, qui deviendra le minitel. Cependant, la traduction du projet d'informatisation sociale français en actions concrètes ne fut pas aussi linéaire que nous venons de le laisser entendre; elle fut aussi marquée par une certaine diversité.

La deuxième période se caractérise par un passage plutôt direct et rapide du projet à la mise au point d'une norme technique (Télidon au Canada et Télétel en France). Mais, elle est aussi marquée par l'amorce d'une réalisation plus incertaine, du moins diversifiée, de la traduction de la norme technique en objets ou système d'objets techno-économiques concrets. En effet, la cristallisation de la norme en produits et services articulés en système cohérent ayant un certain potentiel d'implantation sociale est un processus très complexe qui résulte de l'interaction d'un ensemble d'acteurs (l'Etat et certaines de ses ramifications, des entreprises et des institutions publiques et privées) et de conditions

\footnotetext{
41 Méthode qui consiste à afficher des lettres, chiffres ou diagrammes en agençant des petites unités de couleur.

42 Antiope: Acquisition numérique et télévisualisation d'images organisées en page d'écriture.

43 B. Gurrey et L. Miguet, Guide de la télématique, Paris, Éditions du Centre de formation et de perfectionnement des journalistes, 1990, p. 12-20.
} 
infrastructurelles (la forme de l'État, la position du champ socioéconomique national dans les rapports internationaux, la forme spécifique que prend la crise structurelle dans chaque champ national, l'état des structures industrielles et la taille et la position relative de certains acteurs) d'actions, de réponses à la demande sociale découlant de l'entrée en crise structurelle. Cette interaction complexe détermine les possibilités de conversion du projet en réponse technologique (la norme), puis en propositions concrètes (produits et services) qui la matérialisent dans l'espace socioéconomique national et éventuellement international. Dans cette opération, plusieurs acteurs, plusieurs secteurs, plusieurs logiques industrielles peuvent être et sont impliqués; de sorte que le passage de la norme à un système techno-économique cohérent ayant un potentiel crédible d'implantation peut s'effectuer de diverses façons et susciter différentes et multiples réponses concrètes $^{44}$.

Autrement dit, l'interaction de la multiplicité des acteurs concernés et des conditions infrastructurelles de réponse explique qu'il y ait plusieurs modes de réponse. Cependant, ce sont ces mêmes facteurs qui expliquent aussi que certaines réponses ne réussissent pas à s'imposer (Alex et Antiope), alors que d'autres, promulguées par d'autres acteurs, y arrivent (Télétel) ou semblent en voie d'y arriver (Vidéoway).

Dans la complexité que nous venons très sommairement de décrire, se déroule ainsi, à travers la diversité-multiplicité des acteurs et des propositions, un procès de sélection sociale des acteurs pouvant devenir et devenant porteurs du processus de concrétisation du projet, donc des acteurs détenant un potentiel de structuration. Il ne suffit pas que l'acteur veuille, il faut aussi qu'il puisse. Cependant, cette capacité ne tient pas qu'à sa taille; elle dépend aussi de l'interaction de sa proposition concrète (les produits, services ou systèmes qu'il offre) avec les produits, services et usages sociaux existant déjà. Ainsi, en tenant compte du fait que la radiodiffusion française, au moment où la problématique de l'informatisation sociale se pose, n'offrait que peu de choix (peu de canaux et une programmation restreinte), que la câblodistribution était quasi ou totalement absente du décor télévisuel, que l'ORTF vivait une réorganisation administrative qui l'affaiblissait notablement, qu'elle n'avait pas les capacités économiques de son “concurrent", les PTT, qu'il n'y avait qu'un seul appareil de télévision dans la majorité des foyers, on comprend que l'interconnexion télématique ne pouvait

44 Cette dynamique débouche sur plusieurs réponses originant de secteurs différents et d'acteurs différents. En France, par exemple, les propositions ne sont pas venues que de la téléphonie (Télétel-minitel). L'audiovisuel était aussi partie prenante du "débat" par le biais d'Antiope qui utilisait le téléviseur comme terminal. Au Canada, les télécommunications furent également présentes, entre autres avec le projet Alex de Bell Canada. Dans ce texte, nous ne traitons pas de ces cas de réponses ou de propositions qui ont été secondarisées. Nous l'avons cependant fait dans notre programme de recherche en étudiant les cas d'Antiope et d'Alex. Des rapports sur ces objets sont en cours de rédaction. 
emprunter cette voie. Et, si on tient compte du fait qu'Alex, même s'il se voulait grand public, a surtout été présenté aux milieux professionnels où la téléinformatique était déjà bien implantée, que le grand public n'avait pas l'habitude de se voir facturer en fonction du temps d'utilisation, on comprend que malgré la taille imposante de son promoteur, Bell Canada, il émergeait dans un espace socio-économico-culturel très peu propice à sa survie.

Finalement, nous devons nous rappeler que cette seconde période du processus d'informatisation se caractérise par un changement d'acteur au poste de commande. En France, celui qui prend le relais est directement lié au politique, opère selon une logique de service public et a un pouvoir décisionnel sur les deux secteurs susceptibles de traduire la norme en système concret. Au Canada, les acteurs relais se meuvent dans le marché privé où l'impératif de la survie économique est immédiat et constant. Cela fera qu'ils ne pourront consacrer à la réalisation du projet les mêmes ressources, qu'il y aura une cassure du rythme de réalisation et que la période s'étirera dans le temps (de 1978 à 1983 dans le cas de SID-Télidon, l'ancêtre de Vidéoway, comparativement à 1978-1979 en France). Dès lors, non seulement la filière, l'acteur, la proposition technologique concrète et son degré d'intrégration, mais aussi la trajectoire et le rythme de réalisation du projet vont notablement diverger.

2.3 La mise au point "d'un" prototype et les premières expérimentations sociales (période 3)

Les ressources humaines et techniques investies dès le départ dans le projet télématique français furent beaucoup plus importantes que celles qui le seront au Québec. De plus, la dimension sociétale et politique est présente dès les premiers moments de la réalisation du projet d'informatisation de la société. Au Québec, le caractère proto-marchand va "enfermer" la trajectoire de réalisation dans un dédale d'hésitations et de délais qui vont instabiliser la mise au point d'un prototype et sa "mise à l'essai" sociale. En France, cette période se caractérise par un procès beaucoup plus directif de mise au point technique, d'expérimentation sociale et de formation de la demande.

Dirigée par Philippe Leclercq, un ingénieur en télécommunication, l'équipe de recherche de Télétel $3 \mathrm{~V}$ était composée d'une cinquantaine de professionnels de diverses disciplines (ingénieurs, psychologues, sociologues, graphistes, techniciens, etc.). Cette équipe-projet était divisée en trois sections.

La première se consacrait à la technique. Elle avait comme mandat de construire rapidement un terminal afin que l'expérience grand public puisse commencer à la date prévue, 1981. Pour ce faire, les ingénieurs de Vélizy se sont fortement inspirés du système anglais Prestel; aussi, dans l'expérience de Vélizy, les terminaux servant d'écran de réception étaient des téléviseurs domestiques. 
La deuxième section s'occupait des relations avec les fournisseurs de contenus. Son mandat était de convaincre des entreprises de devenir des fournisseurs de contenus, d'identifier avec ces entreprises le type de services qui pouvaient être développés et d'offrir un soutien professionnel et une aide technique à celles qui acceptaient de s'engager dans l'expérience.

La dernière section était chargée des relations avec les utilisateurs. Ses tâches principales étaient de constituer un échantillon et de faire le suivi des expériences avec les utilisateurs-expérimentateurs afin de saisir les conditions objectives et subjectives d'implantation de la nouvelle technologie.

Outre les expériences menées par les trois sections de l'équipe-projet de Vélizy, il y eut également une expérience publique restreinte (familles et entreprises) réalisée à Saint-Malo. Elle avait comme objectif de tester l'annuaire électronique et un terminal (qui deviendra le minitel). Ce sont les résultats de cette expérimentation qui ont orienté le développement de l'annuaire électronique et de "son" terminal vers la recherche de la rapidité, de la facilité et de la simplicité d'utilisation, qualités qui serviront de base au discours prescriptif que la DGT adressera plus tard aux usagers.

En France, l'offre de la nouvelle technologie de communication s'est donc surtout appuyée sur le développement de l'annuaire électronique effectué en Ille-etVilaine alors que l'offre des contenus télématiques a été élaborée à Vélizy par l'équipe-projet de Télétel 3V. Dans les deux cas, la DGT a dû s'associer avec différents partenaires: l'industrie de l'électronique, pour la construction du terminal $^{45}$ et différentes entreprises privées et différentes institutions publiques, pour l'élaboration des premiers contenus ${ }^{46}$.

L'importance que le projet d'informatisation sociale a pris dans la politique de développement industriel française et la taille économique autant qu'institutionnelle de l'acteur dirigeant la réalisation du projet, les PTT, expliquent le volontarisme de l'implantation, l'impétuosité de l'échéancier de réalisation et l'importance des moyens financiers dont a disposé l'équipe de Vélizy. Tout cela explique aussi que l'équipe-projet ait pu offrir un support important aux entreprises et institutions qui voulurent tenter l'aventure et essayer de devenir des fournisseurs de services. On peut penser que c'est la perspective de devenir un partenaire de la DGT, des PTT, et de participer au projet social le plus important du moment et la possibilité d'avoir accès à un laboratoire grandeur nature, à un échantillon de taille importante, qui a fait que plusieurs entreprises provenant de divers secteurs d'activités ont répondu à l'appel et que la première expérimentation a pu se faire sur la base d'une importante gamme de services (environ 180). Dans cette période du processus de réalisation, bien avant de se faire médiateur entre les fournisseurs

46 3Suisses, La Redoute, la SNCF, l'Éducation nationale, les banques. 
de services et le grand public, l'acteur relais doit donc recruter, animer, organiser les composantes nécessaires à la traduction du projet social en une proposition techno-économique intégrée et concrète.

Si une bonne partie de la base infrastructurelle du procès d'informatisation sociale en France, c'est-à-dire ce que nous avons dit de l'État et de ses liens avec l'acteur relais, explique la rapidité relative de cette cristallisation de l'offre technologique en "une" proposition intégrée, unifié (prototype de terminal et un ensemble de contenus) et relativement stable, une autre partie de cette base rend compte du fait que cela ne s'est pas fait sans problèmes.

En effet, les conditions objectives que connaissent les différents partenaires réels ou potentiels engendrent des contre-tendances ou des résistances plus ou moins actives dues à la concurrence ou à l'indifférence qu'a à surmonter l'acteur principal du processus de réalisation du projet. Ainsi, malgré l'attrait que pouvait avoir l'expérience, l'équipe-projet a dû adopter une stratégie de marketing afin de convaincre les différentes entreprises susceptibles d'être des fournisseurs de services de se lancer dans l'aventure. Mais les coûts encourus par le développement d'un service télématique s'avéraient élevés et relativement prohibitifs pour beaucoup d'entre elles. Aussi, les premiers importants fournisseurs de services furent surtout des entreprises qui avaient une taille et une capacité économique suffisantes pour faire les investissements nécessaires et qui virent dans cette nouvelle technologie un moyen supplémentaire de rentabiliser leurs opérations ou de les rendre plus efficaces. C'était le cas de la SNCF (pour son service de réservations) et des entreprises de vente par correspondance comme La Redoute et les 3Suisses (pour leurs services de commandes téléphoniques).

Cependant, l'obtention de la collaboration de certains acteurs et le soutien de la DGT aux fournisseurs de services dans le développement des services télématiques n'a pas toujours été facile. Ainsi, l'articulation de l'offre en un tout cohérent a aussi été marquée par d'importants conflits dans lesquels la DGT était perçue comme un concurrent. Et ce n'est qu'après une période de conflits (19781980) avec la DGT, que la Presse deviendra un des plus importants fournisseurs de services (services de nouvelles, jeux, services de messageries, etc.) ${ }^{47}$.

Au Québec, on retrouve un processus assez semblable: mise au point d'un prototype, recherche de partenaires et cristallisation d'un produit unifié suffisamment stable pour servir de base à une première expérimentation sociale. Cependant, cette période est plus tardive et le rythme de réalisation est, encore là, plus lent que dans le cas français. Elle va de 1983 à 1986, comparativement à 1980-1981 en France. Tout un ensemble de différences explique cette différence: la taille moins imposante de l'acteur promoteur de la nouvelle technologie, sa

47 Il est intéressant ici, de retenir que le conflit avec la Presse n'a pris fin et que la collaboration active de celle-ci ne s'est manifestée qu'après que la DGT lui eut concédé en télématique, le monopole des petites annonces, de la publicité et des messageries. 
capacité moindre d'attirer, animer et articuler l'action entre les partenaires compte tenu qu'il agit dans un secteur privé de l'économie et que de ce fait il n'avait comme levier pour emporter l'adhésion de partenaires que l'intérêt, pour eux, du lien d'affaire qu'il proposait, la taille beaucoup plus restreinte du marché dans lequel se faisait l'implantation, la logique socioéconomique animant la filière par laquelle elle se faisait (de flot et de club plutôt que d'interconnexion) et la logique socio-économico-culturelle des habitudes des consommateurs des produits émanant de ces filières (de réception et de choix de programmes plutôt que d'échange interactif).

Ce n'est donc qu'entre 1983 et 1986, dans la foulée de son projet SID-Telidon, que Vidéotron mène une série de tests techniques sur son réseau de câblodistribution et entame la construction d'un premier terminal destiné au grand public.

Ces premières travaux sur le terminal n'ont été effectués que par une équipe fort restreinte (cinq ou six personnes) et le premier prototype, baptisé Vidacom, n'a été produit qu'en très peu d'exemplaires ${ }^{48}$.

Parallèlement, Vidéotron s'est associé avec certains partenaires afin de développer la technique elle-même ${ }^{49}$, mais aussi d'élaborer des contenus et des services et de faire une expérimentation sociale de sa technologie ${ }^{50}$.

En 1984, Vidéotron s'associera également avec la compagnie américaine G-Tech, spécialisée dans la construction de terminaux de jeux, et met sur pied une filiale qu'on nommera Vidéoway. Ce partenariat financier, qui n'a duré qu'environ trois ans, témoigne de la volonté de Vidéotron de mettre au point un terminal capable d'offrir une diversité de produits ou de services, du moins un nombre suffisant pour permettre une première expérimentation sociale. Cependant, le niveau encore assez primaire de la technologie proposée par Vidéotron ${ }^{51}$ a rendu la recherche de fournisseurs de services plutôt ardue. Très peu d'entreprises ont vu alors dans cette nouvelle technologie un potentiel réel de nouveau média. De plus, parce que l'expérience grand public envisagée par Vidéotron ne comportait que 500 foyers, les entreprises sollicitées ont eu tendance à estimer que l'investissement en temps et en argent était trop important pour les avantages qu'elles pouvaient en

48 Une centaine d'appareils ont été fabriqués (entrevue avec un ingénieur de Vidéotron).

49 Avec les universités de Sherbrooke et de Montréal (Polytechnique), le Centre de recherche industrielle du Québec (CRIQ) et la Société Micro Électronique de Sherbrooke.

50 Avec le professeur Michel Cartier du département de communication de l'Université du Québec à Montréal.

51 Pour l'époque, la technologie proposée était indiscutablement une nouveauté. Cependant, la capacité relativement faible du prototype et des vecteurs de transmission (des câbles) a fait apparaître l'innovation de Vidéotron comme une traduction peu crédible du projet informatique, ce qui a rendu fragile et a relativement délégitimé son discours visant à faire accepter sa technologie et à recruter des partenaires. 
espérer. Vidéotron ne commencera donc sa première expérimentation grand public qu'avec seulement cinq ou six sortes de services. Toutefois, comme dans le cas français, il y avait d'importants partenaires: Hydro-Québec et Loto-Québec. Mais, contrairement au cas français, ces partenaires étaient très peu nombreux; et ils le demeurent encore aujourd'hui.

Comme on peut le constater, la troisième période du processus d'informatisation sociale se caractérise par une complexification notable. À la fixation-stabilisation de la technologie dans un prototype s'ajoute l'incorporation de contenus également prototypes. L'acteur promoteur doit donc dans cette opération s'associer avec divers acteurs œuvrant dans des secteurs connexes et complémentaires (pour en faire des partenaires) ou s'y substituer lorsqu'ils ne se manifestent pas en tant que collaborateurs ou encore les concurrencer, ce qui implique une certaine socialisation du processus de construction de l'offre et de son introduction sur le marché. On arrive enfin à concrétiser l'offre technologique par quelque chose de tangible, d'objectivé, un terminal, qui peut être soumis à l'expérimentation sociale. Ces premières expériences font entrer le processus dans une phase d'ajustements de l'offre, non plus cette fois à partir des contraintes techniques (la réalisation d'un objet technique tangible qui fonctionne), mais à partir de la contrainte stratégique dans l'implantation sociale, la réaction du public. C'est à ce moment que les consommateurs entrent en tant qu'acteurs réels, c'est-àdire actifs et non plus seulement comme image-prétexte aux volontés de développement de l'offre, dans le processus d'informatisation sociale et que commence un certain "dialogue" entre l'offre et le public ${ }^{52}$. Cette amorce se fait toutefois sur la base d'une offre encore "non terminée" qui devra être ajustée.

L'entrée en scène du public est d'une importance capitale dans le déroulement du processus, car, selon les réactions du public, ce ne sont pas seulement la technique et les contenus qui seront ajustés, remaniés, développés. En effet, s'enclenche alors la construction d'une représentation sociale ${ }^{53}$ de la technologie et des objets et services qui la concrétisent, mais aussi des acteurs en présence et de

52 Ici, à dessein, et cela est significatif sur le plan conceptuel, nous n'employons pas le terme demande.

53 Il est évident que le processus de construction de la représentaiton sociale des NTIC commence dès les premiers moments du processus d'informatisation avec la formulation du projet de société informatisée et la présentation de ce projet à la société par la publication de rapports de commissions d'enquête sur la question, la couverture de presse, les déclarations publiques des décideurs politiques et économiques et des experts de toute sorte. Il s'agit toutefois, à ce moment, d'une représentation fondamentalement "idéologisée" et essentiellement marquée par l'idéalisme et l'utopisme. Au fur et à mesure que s'ajoutent et s'articulent certaines réalisations, le projet acquiert le caractère d'une réalité concrète, incontournable, objective. C'est à ce moment que la représentation se fait "concrète", "vraie", et qu'elle constitue un véritable levier de persuasion pour le discours prescriptif. 
leurs rapports ${ }^{54}$. De fait, il s'agit de construire une image objectivant non seulement les objets informatiques, mais surtout le consommateur-demandeur en mettant en correspondance l'offre avec "quelque chose" qu' on nommera "besoin de la nouvelle technologie" et qui légitimera l'antériorité de l'offre en "normalisant" la présence du consommateur en le situant dans un rapport d'égalité avec l'offre, en l'inscrivant dans un rapport dialogique, comme l'a nommé Pierre Mœglin ${ }^{55}$.

Les responsables de cette construction furent nombreux. Nous avons déjà souligné le rôle de l'État et des promoteurs. Il faut ajouter les médias de masse et l'école. Mais toute cette activité de construction-transmission de la représentation ne peut se faire que sur la base de l'objectivation du projet, des objets technoéconomiques le concrétisant, des acteurs et de leurs rapports. Ce sera là le rôle de la recherche.

Cette période du processus est donc aussi marquée, à des degrés divers selon les situations nationales et les acteurs promoteurs-animateurs du processus, par la prolifération de la recherche sociale sur l'informatisation, dans laquelle les descriptions et les analyses des réactions du public, des "usages" et des usagers, ont occupé une place de premier plan, et continuent encore de le faire. L'effervescence de la recherche ${ }^{56}$, le support de l'État et des entrepreneurs dont elle a bénéficié, et l'orientation que ces "commanditaires" lui ont imprimé montrent la centralité de son rôle à ce moment ${ }^{57}$.

Finalement, à la fin de cette troisième période du processus, on peut considérer que sont en place toutes les composantes faisant du processus d'informatisation un procès social. L'acteur relais, promoteur et animateur de la mise en place de l'offre a alors réussi à mettre au point une proposition intégrée et unifiée. Le public est impliqué dans le processus par le biais des expérimentations sociales et la représentation qu'on en donne. La solution informatique prend alors une place concrète dans la dynamique sociale. Cette place est cependant fort réduite, mais le rassemblement des composantes va constituer la base "objectivée" et légitimatrice des efforts de son élargissement. L'implantation sociale comme telle peut alors commencer.

54 C'est aussi à ce moment qu'une bonne partie du discours social sur les nouvelles technologies d'information et de communication se fait idéologie aliénante en cachant, sous le masque de l'offre-demande, la position réelle des acteurs sociaux en interaction dans le processus d'informatisation.

55 P. Mœglin, "Télématique: De la recherche sur les usages aux usages de la recherche", article cité, p. 43 et suiv.

56 Voir à ce sujet, TIS-Réseaux, Dix ans de vidéotex, vol. 2, no 1 et novembre 1989, no 37.

57 Voir à cet effet, P. Mœglin, ibid.; également, J.-G. Lacroix et B. Lévesque, "Principaux thèmes et courants théoriques dans la littérature scientifique en communication au Québec", Communication, vol. 7, no 3, p. 183 et suiv. 
2.4 L'amorce de la socialisation de la nouvelle technologie: le début de l'implantation grand public (période 4)

En France, cette quatrième étape du processus, 1981-1982, correspond à la dernière période de l'expérience de Télétel $3 \mathrm{~V}^{58}$. C'est à ce moment que la troisième section de l'équipe-projet constitua un échantillon important (environ 2 300 foyers) et que fut mis au point un protocole d'installation et de démonstration pour permettre aux utilisateurs expérimentaux de se familiariser avec la nouvelle technologie. Cette expérimentation a permis à la DGT et aux fournisseurs de services d'ajuster leur offre, c'est-à-dire de terminer la mise au point d'une offre qu'on voulait voir être l'objet de "certaines utilisations" de la part, surtout, $d$ 'un grand nombre d'utilisateurs.

Cela s'est entre autres fait grâce à des réunions d'usagers organisées par l'équipe-projet, rencontres qui permirent d'évaluer les réactions du public. Cela a amené certaines entreprises à abandonner le développement des services télématiques qu'elles proposaient parce qu'il ne semblait pas y avoir d'utilisations effectives, alors que d'autres ont pu les remanier ou les ajuster. Très peu de services furent d'emblée acceptés et adoptés par les usagers expérimentateurs. On ne saurait trop souligner l'importance du fait que c'est le promoteur qui a mis en branle le processus d'ajustement social de son offre technologique unifiée et intégrée et, ainsi, la médiation entre le public et les fournisseurs de services, entre ce qui était proposé et les utilisations effectives que cela suscitait ${ }^{59}$. Cela montre bien que l'antériorité de l'offre demeure encore à ce stade du processus. Le rôle de l'équipe-projet durant cette période l'indique sans équivoque; et le cas du développement des messageries ne fait pas exception à cette "règle".

En effet, c'est à partir d'un service de boîte aux lettres qui n'intéressait pas les utilisateurs expérimentateurs et à la suite des critiques et suggestions de ces derniers recueillies par l'équipe-projet, que ce service fut transformé en club social électronique grâce auquel les utilisateurs pouvaient converser sur un sujet qui les intéressait. Ce n'est qu'après cet ajustement que la prolifération des messageries s'enclencha. Ainsi, lorsqu'on prend en compte la portée structurante de la médiation effectuée par le promoteur animateur de la mise en place de l'offre dans l'ajustement de l'offre, on est forcément amené - contrairement à ce que soutient

58 Notons que Télétel 3V a constitué, dans les faits, beaucoup plus un projet pilote qu'une expérience, et qu'elle n'a pas connu d'évaluation comme telle ni de fin officielle. On a décidé d'un élargissement et on a "tout simplement" commencé à en parler au passé.

59 Ce qui implique toute la gamme des réactions réelles, effectives, de l'accueil acceptation apologétique à l'implication individuelle enthousiaste et innovatrice (les bricoleurs) et aux utilisations plus ou moins conformes ou déviantes de celles prévues, suggérées ou recommandées par les promoteurs, en passant par la sous-utilisation (l'utilisation d'une partie des capacités des systèmes proposés), l'indifférence, et, finalement, le refus pur et simple, voire obstiné. 
tout un courant de la littérature sur les usages - à relativiser le poids "créateur", structurant, du moins à ce moment, des usagers dans le processus de mise en place des NTIC.

Par ailleurs, au cours de l'expérience de Vélizy, l'implantation a été notablement élargie, le nombre des terminaux installés ayant augmenté de $30 \%$. Cet élargissement semble toutefois s'être beaucoup plus fait dans la direction des fournisseurs de services $(+97,9 \%)$ et de la visibilité sociale de la technologie, la promotion $(+149,3 \%)$, que vers les utilisateurs privés, les ménages $(+4,2 \%)$. De plus, durant cette période, les fournisseurs de services ont manifesté un intérêt grandissant pour le développement de services professionnels. Il semble donc qu'il y ait eu une certaine "dérive" de l'intention d'implantation, ou du moins un certain glissement concernant la population cible de l'implantation vers les usagers professionnels.

Ainsi, dans ce cas, l'affinement de l'offre par la mise à l'essai sociale, l'expérimentation grand public, débouche sur un réalignement de la trajectoire de réalisation, ce qui nous permet de constater la complexité du processus de mise en place de l'offre et l'incertitude qui règne sur l'enclenchement de la dialectique offredemande, même quand le projet social est précis et que la trajectoire de réalisation est directive et le plus linéaire possible.

Au Québec, le déroulement du processus dans cette quatrième période est tout aussi complexe, mais il ne donne pas lieu à la même dérive en ce qui concerne la population cible de l'opération d'implantation sociale.

En 1986, Vidéotron rachète les actions de G-Tech afin d'être l'unique propriétaire de la technologie Vidéoway. La même année commence la première expérimentation grand public. Échelonnée sur trois ans, 1986-1989, cette expérience est menée en collaboration surtout avec Hydro-Québec et Loto-Québec dans 500 foyers de la ville de Brossard (rive-sud de Montréal). Comme dans le cas français, cette expérimentation a permis de faire avancer la mise au point de la technologie ${ }^{60}$ et de réaliser une première étude des réactions grand public.

Cependant, le nombre restreint de services $^{61}$ et l'état primitif de la bidirectionnalité62 rendaient la technologie proposée peu attrayante autant pour les

60 Le terminal utilisé au cours de cette première expérimentation était à peu de choses près identique à l'actuel terminal et il avait, à l'exception de la télévision interactive, la même capacité de transmission. Il constituait la quatrième génération du terminal Vidéoway.

61 Les services alors offerts étaient la gestion de l'énergie, un décodeur pour la télévision payante et la télématique, des logiciels de jeux et un service de nouvelles.

62 La bidirectionnalité de la technologie alors proposée par Vidéotron nécessitait l'utilisation d'un réseau parallèle, le téléphone. C'est encore le cas aujourd'hui. Quant à l'interactivité, elle était fort réduite et dépendait d'un télédéchargement préalable dans 
éventuels fournisseurs de services que pour les usagers, ce qui a coupé court à la prétention du promoteur d'avoir mis au point un nouveau média plus performant. C'est sans doute ce qui a amené Vidéotron à créer, en 1988, une nouvelle filiale, Vidéotron Plus, dont le mandat sera assez semblable à celui de la seconde section de l'équipe-projet de Vélizy, c'est-à-dire trouver des fournisseurs de services et travailler à élaborer des contenus ${ }^{63}$.

À l'automne 1989, Vidéotron juge suffisante la gamme des services offerts pour se lancer dans une opération de commercialisation de sa technologie et de son terminal Vidéoway. Cependant, une deuxième expérimentation grand public est enclenchée, cette fois avec un terminal identique à celui qui servira de base à la première phase de commercialisation qui débutera en 1990 (période suivante). Pour Vidéotron, il ne s'agit toutefois pas d'une vraie expérience, mais plutôt d'un prélancement grand public et publicitaire.

Cette deuxième expérimentation - menée dans la ville de Longueuil et dans une partie de la ville de Brossard auprès de 350 foyers - sera plus courte que la première; elle se fera d'octobre 1989 à mars 1990 en collaboration avec le professeur André Caron du département de communication de l'Université de Montréal. Elle visera essentiellement à réaliser un profil des utilisateurs types et des pratiques effectives, et à cerner la satisfaction vis-à-vis du système à implanter ${ }^{64}$. Le fait que le cadre de cette recherche universitaire ait fortement été influencé par la filiale Vidéotron Plus nous amène à penser que cette dernière expérimentation avait surtout comme objectif, du moins dans l'esprit de l'entreprise qui la soutenait, de préparer le discours prescriptif qui devait accompagner la première phase de commercialisation. Finalement, peu de temps après le démarrage de cette seconde expérience, Vidéotron estime que le moment est venu de lancer sa première opération de commercialisation grand public. Ce qui ouvre une autre période du processus, le début de l'implantation générale.

La quatrième période du processus d'informatisation se déroule donc sous le sceau de l'affinement et de la diversification de l'offre. Mais, ces ajustements se

le terminal domestique. C'est encore le cas aujourd'hui. Comme on peut le constater, on est encore fort loin d'une véritable télématique interactive, laquelle nécessite, rappelons-le, une interconnexion universelle point à point en temps réel. Toutefois, au moment où ces lignes sont écrites, Vidéotron annonce une deuxième phase d'interactivité, que l'entreprise nomme la bidirectionnalité. Voir à ce propos V. Beauregard, "Vidéoway prend pied en Ohio", La Presse, 30 octobre, 1993, p. F-1.

63 G. Gagnon, K. Vibert, S. Laborde-Kastex et J.-G. Lacroix, Télématique et télévision interactive, des industries en mutation. L'informatisation du processus de travail: le cas de Vidéotron Plus et de TVI, Montréal, GRICIS, 1993.

64 A. Caron et al., Analyse de l'adoption d'une nouvelle technologie, le systèeme Vidéoway, Montréal, GRJM, département de communication, Université de Montréal, 1992; voir également, P. Bélanger, Le système Vidéoway. Modalités d'adoption d'un système interactif de télévision, thèse de doctorat, Montréal, département de communication, Université de Montréal, 1991. 
font alors en fonction de leur insertion sociale. Ils sont ainsi conditionnés par l'utilité ou l'inutilité sociale qui se révèle par les utilisations effectives, les résistances, les non-utilisations, voire le refus.

Du côté de l'offre, la complexification se poursuit. Le promoteur initiateur de l'offre technologique se fait aussi organisateur d'un ensemble grandissant de contributions et de partenaires. Cette articulation de l'offre en un tout intégré se fait toutefois de façon fort différente selon les situations socioéconomiques nationales. Dans le cas français, les collaborations complétant l'offre technologique sont extérieures à l'entreprise promotrice, ce qui affirme beaucoup plus son statut d'animateur de l'offre et donne à la structuration de celle-ci un caractère nettement plus complexe, socialisé. Par ailleurs, cela rend sa position d'intermédiaire entre le public et les fournisseurs de contenus, de médiateur, plus évidente dès l'enclenchement de l'expérimentation sociale. Dans le cas québécois, même s'il y a bel et bien une certaine recherche de partenaires pour le développement de services, la grande majorité des propositions à ce niveau vont venir de l'entreprise promotrice elle-même ou de ses filiales. Cette stratégie, Vidéotron l'a déjà utilisée au moment où il lui était nécessaire de multiplier les canaux afin de développer son marché et ainsi renforcer sa position dans la structure de l'industrie télévisuelle ${ }^{65}$. Dans le cas français, la partie contenu de l'offre est mise en place par des acteurs déjà présents et souvent importants dans un ensemble diversifiés de secteurs (presse, grands magasins, chemin de fer, banques...). Cette mise en place de l'offre relève donc, malgré certains conflits initiaux, de l'interaction intersectorielle. Ainsi, la nouvelle technologie devient, potentiellement à ce moment du processus, un facteur de réarticulation industrielle intersectorielle, ce qui accrédite la technologie proposée comme traduction concrète du projet de société informatisée et réponse unifiée et crédible à la crise structurelle. Au Québec par contre, la mise en place de la partie contenu de l'offre relève davantage de la substitution ou de la suppléance par l'acteur promoteur de la technologie, de sorte que la diversification découle plutôt du développement de l'organisation du travail à l'intérieur de l'entreprise ${ }^{66}$. Cela n'a évidemment pas le même potentiel de réarticulation industrielle intersectorielle et, donc, n'a pas le même potentiel de concrétisation du projet social informatique.

Cette différenciation dans le processus de mise en place de l'offre selon les contextes nationaux et les technologies étudiés ne tient pas qu'à la différence des filières d'où émergent les propositions technologiques, à la nature de l'offre et à la stratégie des entreprises promotrices. Elle tient aussi aux conditions infrastructurelles, particulièrement la densité industrielle (le nombre et la taille des

65 Voir à ce sujet, G. Tremblay et J.-G. Lacroix, Télévision. Deuxième dynastie, op. cit., p. 127, particulièrement la note 60 .

66 G. Gagnon, K. Vibert, S. Laborde-Castex et J.-G. Lacroix, Télématique et télévision interactive, des industries en mutation. L'informatisation du processus de travail: le cas de Vidéotron Plus et de TVI, op.cit. 
joueurs) dans les secteurs touchés ou impliqués par le processus et la taille du marché.

Du côté de la "demande", le public devient un acteur, qui, toutefois, n'a qu'un potentiel structurant fort restreint parce que limité à la mise à l'essai sociale de l'offre, donc relégué à un rôle de "cobaye" utilisé afin d'ajuster l'offre. Cependant, cette interaction permet au promoteur de l'offre intégrée (technique et contenus) de la présenter comme la réalisation du projet social qui répond à un besoin puisqu'elle subit avec succès l'épreuve de l'adoption sociale, en témoigne l'expérimentation sociale. De ce moment, le processus entre dans un mouvement de généralisation et le discours prescriptif des promoteurs acquiert une importance déterminante. C'est alors que commence "vraiment" le processus de formation des usages sociaux de la nouvelle technologie.

2.5 La première phase de commercialisation: l'atteinte de la masse critique de légitimation sociale (période 5)

En février 1990, Vidéoway est lancé sur le marché montréalais. On le présente comme un système de télécommunication vidéonumérique grand public résultant de l'intégration de plusieurs services: câblodistribution, télévision payante, télévision interactive, télégestion des services aux abonnés, sous-titrage pour malentendants, télétexte, vidéotex, jeux vidéo et courrier électronique ${ }^{67}$.

La première action d'implantation générale du Vidéoway a consisté à imposer à tous ses abonnés à la télévision payante (TVP) le nouveau terminal. Du jour au lendemain, grâce à la numérisation du signal, ces abonnés ont donc constitué la masse critique des premiers adhérents, laquelle va servir de base légitimatrice au discours prescriptif et publicitaire du promoteur présentant sa télématique audiovisuelle comme le futur, la modernité, l'incontournable moyen d'entrer dans le 21 e siècle et dans l'ère de la société de l'information et une offre socialement pertinente car elle connaît du succès ${ }^{68}$ puisque "déjà de nombreux consommateurs l'adoptent ${ }^{69}$ ".

67 Selon un dépliant promotionnel de Vidéoway ltée, 1990.

68 Alors, en 1990, le nombre d'abonnés s'élevait à 30000 , soit 3,31\% du nombre total d'abonnés de Vidéotron. À ce moment, il y avait environ 1,6 million abonnés à la câblodistribution au Québec; ainsi, la proportion de consommateurs "adoptant" la technologie proposée ne représentait que de 1,86\% de l'ensemble des abonnées québécois. Sources: Le Groupe Vidéotron, Rapport Annuel, différentes années; V. Beauregard, "Vidéoway prend pied en Ohio", La Presse, 30-10-1993, p. F-1; ministère des Communications du Québec, Profil des industries de la câblodistribution, de la télévision payante et des canaux spécialisés au Québec: 1987-1990, Québec, mai 1992.

69 P. Rigaud et J.-G. Lacroix, Vidéotron-Vidéoway. Discours promotionnels et prescriptions d'usages, op. cit. 
Parallèlement, avec le remplacement d'autorité des vieux décodeurs, Vidéotron lance une vaste campagne publicitaire et de marketing qui, en plus d'augmenter graduellement le nombre des abonnés à la nouvelle technologie, la rend visible sur la place publique et l'impose comme une réalité inévitable et souhaitable, "merveilleuse 70 ".

Cette réalité ne prend toutefois corps qu'inégalement et graduellement, par secteurs géographiques, au fur et à mesure que l'approvisionnement en terminaux le permet $^{71}$ et que les centrales informatiques régionales du promoteur sont modernisées afin de permettre la vidéocompression ${ }^{72}$ des signaux grâce à laquelle l'offre de contenus peut être très substantiellement élargie (passage de 35 à environ 80 canaux et éventuellement à plus de 100).

En août 1991, le nombre des abonnés à Vidéoway atteignait environ 103000 , ce qui représentait alors $10,95 \%$ des abonnés de Vidéotron et $6,16 \%$ du nombre total des abonnés québécois au câble ${ }^{73}$. La pénétration de la nouvelle technologie restait donc modeste, malgré les campagnes publicitaires qui se succédaient. De plus, Vidéotron n'est pas arrivé à stabiliser ses acquis; les débranchements d'abonnés à Vidéoway furent et demeurent nombreux ${ }^{74}$. L'implantation piétine donc et est encore fragile. De toute évidence, la campagne de séduction orchestrée par le promoteur n'a pas soulevé, comme ce fut le cas pour le magnétoscope, une vague impétueuse d'adhésions. Cela questionne autant la "qualité" de la

70 G. Gagnon, J.-G. Lacroix et G. Tremblay, Historique de Vidéoway, op.cit.

71 En 1990, les terminaux Vidéoway étaient fabriqués par la compagnie Zenith de Chicago dans une de ses usines au Mexique. Les livraisons étaient inégales et irrégulières (entrevue avec un coordonnateur de marketing chez Vidéotron).

72 La vidéocompression consiste à comprimer un signal vidéo afin qu'il prenne moins de place dans un canal transporté par câble. On peut ainsi livrer quatre, cinq, six... canaux à l'intérieur d'un canal d'un câble actuel (coaxial-cuivre) ou prochain (optique). C'est ce que les câblodistributeurs appellent le multiplexage. Cette compression constitue toutefois une surcharge qui nécessite une amplification notable du signal. Il faut également des câbles pouvant supporter cette intensité accrue des signaux. Ainsi, pour passer à 78 canaux NTSC (National Television System Commitee), selon le comité qui a donné son nom à cette norme, il faut atteindre environ les 550 mégahertz. Dans l'industrie on estime que la capacité maximale des câbles coaxiaux est d'environ 150 canaux NTSC. Au-delà de ce nombre de canaux, ce qu'on peut nommer "l'enveloppe technique" du câble de cuivre (sa limite extrême) est dépassée, défoncée; une autre enveloppe technique est nécessaire. C'est là que la fibre optique devient incontournable. Voir à ce propos, CANADA, Convergence: concurrence et coopération. Politiques et réglementation concernant les réseaux locaux du téléphone et de la câblodistribution, Ottawa, Ministère des approvisionnements et services Canada, 1992, p. 24.

73 Sources: voir la note 68 .

74 Au cours de la première campagne (automne 1991), 62000 foyers se sont abonnés à Vidéoway. De ce nombre, 19000 se sont par la suite débranchés (entrevue avec un coordonnateur du marketing chez Vidéotron). 
technologie proposée (la bidirectionnalité et l'interactivité du système proposé) que l'utilité sociale qu'y voient les consommateurs dont la demande solvable, payante est en ce moment faible ${ }^{75}$. Il n'est donc pas surprenant que les critiques se firent assez nombreuses au sujet des coûts en investissements, du prix de revient pour les citoyens et des avantages et des progrès réels que constituait la nouvelle technologie.

La première phase de commercialisation du système Vidéoway a donc plafonné assez rapidement ce qui a placé le promoteur dans une situation relativement difficile, du moins quant à son image de marque et à son succès. Cela l'a conduit à recourir, encore une fois avec la bénédiction du CRTC (voir la décision de juin 1993 concernant l'adressabilité universelle que nous définirons plus loin), à la coercition pour faire un pas en avant en forçant, cette fois-ci, la masse des abonnés à accepter son offre. Ce qui amorce la dernière période du processus, la généralisation.

Ce mélange de coercition et de séduction dans l'opération de généralisation se retrouve également dans le cas français durant cette cinquième période du processus.

L'objectif fondamental du projet télématique français était de développer un système grand public. Compte tenu de la dérive concernant la population cible sur laquelle se termine la phase d'expérimentation sociale (la quatrième et précédente période) trois choix s'offrent au promoteur pour réaliser une première phase de généralisation: 1) élargir au grand public l'expérience de Vélizy même si l'opposition de certaines entreprises de presse, malgré la fin du conflit avec la DGT, percevaient encore la nouvelle technologie comme un concurrent et exerçaient une certaine pression pour en freiner le développement à grande échelle; 2) restreindre l'utilisation du minitel à l'annuaire électronique; 3 ) offrir au grand public l'accès à la télématique par le biais de l'annuaire électronique et faire commencer les autres services par des applications professionnelles. C'est cette dernière stratégie qui a été retenue.

Lors du lancement commercial de sa nouvelle technologie, la DGT présente le minitel et l'annuaire électronique comme les remplaçants de l'annuaire papier, mais les différents services télématiques élaborés pendant l'expérience de Vélizy ne sont pas publicisés. Aussi, la première phase d'implantation générale se fait-elle selon une offre assez restreinte.

75 En 1992, le nombre des abonnés à Vidéoway s'élevait à 167751 , ce qui représentait $17,39 \%$ du nombre des abonnés québécois de Vidéotron. Cela représentait $9,74 \%$ de tous les abonnés québécois au câble. Cependant, le nombre des abonnés à la télévision payante chez Vidéotron était de 156577 , ce qui représentait 93,3\% des abonnés à Vidéoway. Parce que l'accès à la TVP requiert l'utilisation du terminal Vidéoway, cette donnée nous amène à penser que sans cette coercition, très peu d'abonnés "choisiraient" d'adopter la nouvelle technologie "proposée" par le promoteur. 
Toutefois, le rythme de l'approvisionnement a obligé la DGT à ne distribuer le minitel que région par région. La télématisation du territoire ne se fait donc qu'inégalement, par plaques régionales, et va s'étendre sur une certaine période. Ce qui brise la cadence accélérée de réalisation du projet. De plus, en 1983, la France vit à l'ère de la décentralisation, ce qui force la DGT à ajuster sa stratégie d'implantation grand public. Plutôt que d'imposer le minitel aux régions, elle va faire en sorte que ce soient elles qui le demandent.

La stratégie du promoteur de l'offre a toutefois été beaucoup plus contraignante qu'elle n'apparaît de prime abord. En effet, pour faciliter l'implantation domestique du minitel, la DGT l'a offert gratuitement et l'a présenté comme le remplaçant de l'annuaire papier, tout en laissant entendre que ce dernier devait être retiré, ce qui a soulevé bon nombre de protestations et amené l'offreur promoteur à freiner ses velléités coercitives et à adopter une tactique plus subtile.

Par ailleurs, on a laissé aux fournisseurs de services le soin de mettre au point des applications et de les promouvoir. Cette période fut ainsi marquée par un abandon élevé et rapide des essais. Elle se caractérise aussi par de grands succès. Certains services, comme les programmes de révision du baccalauréat et les résultats aux examens, furent utilisés très massivement. Cependant, le succès même de ces services a fait qu'ils ne purent devenir rentables parce qu'ils nécessitaient de fortes dépenses pour être mis en place et opérés alors que leur utilisation ne se faisait qu'irrégulièrement et durant une période restreinte et très circonscrite.

Ainsi, ce sont les exigences de la rentabilité économique qui semblent avoir freiné en France l'implantation d'une certaine télématique, c'est-à-dire de services qui étaient techniquement réalisables et qui faisaient l'objet d'utilisations massives. Ce sont aussi ces contraintes qui vont par la suite orienter le développement et donc la diversification des applications autant que le rythme et les cibles de l'implantation et les types d'usages qui seront privilégiés par l'offre et promulgués par le discours prescriptif.

Finalement, durant cette période, la DGT continue de remplir son rôle de médiateur, entre autres, en faisant un suivi auprès des utilisateurs par le biais de sondages appelés MV2. Un autre type de suivi fut effectué auprès des fournisseurs de services par le maintien d'une structure sectorielle, c'est-à-dire des ingénieurs commerciaux affectés à chacun des secteurs importants (banques, presse, agriculture, etc.).

La cinquième période du processus et première phase de généralisation ou de commercialisation est caractérisée par un amalgame de contraintes et de séductions prescriptives. La dialectique des deux faces de cette opération (coercition et 
séduction) va permettre de recruter suffisamment d'utilisateurs ${ }^{76}$ pour constituer, à deux niveaux, une masse critique. D'une part, le rassemblement d'une certaine masse d'utilisateurs objective socialement les objets techniques utilisés et les utilisations. Autrement dit, si les utilisations des NTIC durant cette période ne peuvent être qualifiées d'usages sociaux véritables parce qu'elles ne sont pas encore organiquement intégrées et récurrentes dans la vie quotidienne des citoyens en général, elles n'en ont pas moins, du fait de cette masse, un poids social bien concret en devenant visibles et perceptibles par les utilisations elles-mêmes et par les acteurs de ces pratiques et non plus seulement par le discours prescriptif et en bousculant et questionnant, ainsi, les pratiques parmi lesquelles elles viennent s'insérer. D'autre part, cette masse critique permet aux promoteurs des NTIC de croire au succès de l'implantation et de le "claironner". Elle renforce donc et légitimise la base coercitive (violente) du discours de séduction prescriptive ${ }^{77}$. Même si l'implantation plafonne à un moment donné, l'atteinte de cette masse critique permet aux promoteurs de se lancer dans la "vraie" généralisation.

\subsection{Vers la généralisation de la nouvelle technologie (période 6)}

Le moment et le contexte où s'enclenche la sixième et "dernière" période sont fort différents dans les deux cas que nous avons étudiés. Dans le cas de Télétel, la généralisation émerge dès 1983-1984 (1993-1994 pour Vidéoway), ce qui peut s'expliquer par la nature et la maturité de la technologie Télétel (sa cohérence et sa stabilité relative comme système diversifié et intégré), la place de l'acteur promoteur dans le tissu industriel français et celle du projet d'informatisation sociale dans la politique de développement économico-technique de l'État français. Dans le cas de Vidéoway, la généralisation apparaît beaucoup plus tard, mais elle surgit dans un contexte de développement accéléré de la technologie informatique, de forte concurrence entre plusieurs industries qui, avec la généralisation de la numérisation, se voient confrontées à la perspective de la production et de la distribution de produits et services numérisés intégrés (son-image-texte) requérant de nouveaux vecteurs de transport et de nouveaux équipements de consommation, d'utilisation, de l'information. C'est donc un contexte où plusieurs acteurs sont simultanément confrontés à des marchés qui semblent tendre à s'unifier ou du moins à entrer dans une phase de convergence.

76 Qu'on qualifiera dans le discours prescriptif, publicitaire, de Vidéotron de “pionniers". P. Rigaud et J.-G. Lacroix, Vidéotron, Vidéoway-Vidéoway. Discours promotionnels et prescriptions d'usages, op. cit.

77 Partie du discours sociale constituée d'assertions laissant entendre que ne pas adopter la nouvelle technologie c'est ne pas adhérer au progrès, c'est rater le train de l'histoire, c'est ne pas donner toutes les chances à ses enfants, c'est retarder l'avènement du futur, etc. Ibid. 
La stratégie de Vidéotron et de ses associés câblodistributeurs québécois et canadiens visant à obtenir du CRTC l'adressabilité universelle ${ }^{78}$ n'avait pour but que de se mieux positionner dans ce marché en formation par rapport aux concurrents d'autres industries, particulièrement les télécommunications et la diffusion directe par satellite. Cette adressabilité va probablement procurer aux câblodistributeurs canadiens un revenu supplémentaire très substantiel ${ }^{79}$, ce qui leur permettra de renforcer structurellement leur position concurrentielle dans le contexte de convergence.

Cependant, cette généralisation de la proposition techno-économique de Vidéotron et de ses associés n'est pour le moment que potentielle. Beaucoup de choses restent à déterminer et à mettre en place. Il s'agit donc d'un horizon qui renferme encore beaucoup d'inconnues, d'incertitudes, entre autres à propos du temps qui sera nécessaire à sa réalisation, au sujet de la forme concrète que la technologie prendra, particulièrement au niveau de l'intégration et de la diversification des contenus, à propos des réactions des usagers. Toutefois, le flou et l'indétermination qui règnent sur la traduction du projet "Vision 200180" en réalité technique et sociale concrète, implantée et généralisée, ne rend pas pour autant le processus complètement indéterminé, tellement chaud — pour reprendre le vocabulaire de Callon et Latour ${ }^{81}$ - qu'il en devienne vaporeux et insaisissable. On sait des choses. Et, grâce à ces connaissances, on peut entrevoir le faisceau des

78 L'adressabilité universelle est une technique de transport de signaux qui permet de livrer dans un foyer particulier un programme spécifique choisi par un consommateur. On la dit universelle parce qu'elle doit permettre de livrer une information (audio et vidéo) dans n'importe quel foyer d'un territoire desservi par un câblodistributeur.

79 Selon nos estimations, Vidéotron pourrait retirer en sept ans, soit la durée du scénario présenté au CRTC, autour de 700 millions de dollars additionnels. J.-G. Lacroix, G. Tremblay, M. Ménard et M. Saint-Laurent, Télévision 2001: câblodistributeurs et étoiles de la mort, texte de la communication présentée au congrès annuel de I'Association canadienne de communication tenu à Ottawa en juin 1993, Montréal, GRICIS, 1993, p. 12.

80 L'étude intitulée A Financial and Regulatory Analysis of the Canadian Cable Television Industry Too the Year 2001, Toronto, décembre 1992, réalisée par la firme Peat, Marwick, Stevenson et Kellogg pour l'Association canadienne des câblodistributeurs a servi d'assise à prétention scientifique à ces derniers pour faire pression sur le CRTC. Elle fait état de trois scénarios possibles pour la période de sept ans allant de 1994 à 2001. Sauf l'affirmation de la nécessité de l'adressabilité universelle pour assurer la survie de l'industrie de la câblodistribution au Canada appuyée sur une analyse économique très classique, mais menée sur la base d'hypothèses très contestables, particulièrement à propos du potentiel de substitution de la diffusion directe par satellite - elle demeure plutôt vague sur les réalisations concrètes. Ainsi, même au niveau du projet, la démarche se caractérise par beaucoup d'incertitude.

81 M. Callon et B. Latour, "Les paradoxes de la modernité. Comment concevoir les innovations?", Prospective et santé, no 36, hiver 1985, p. 13-25. 
probabilités qui orientent la "concrétisation" du devenir social de l'informatisation, du moins dans la version Vidéotron-Vidéoway.

On sait, entre autres, que l'autorisation accordée aux câblodistributeurs canadiens par le CRTC va notablement consolider leur position concurrentielle dans le contexte de la convergence parce qu'en plus de leur permettre d'amortir très rapidement leurs dépenses pour la modernisation technologique de l'industrie, de pouvoir ainsi maintenir, si ce n'est augmenter, leur taux de rendement et d'accroître leur capitalisation, cela va les faire apparaître comme les détenteurs de l'offre concrétisant le projet social informatique. On sait, par ailleurs, que le renforcement économique de leur position peut conduire à des alliances d'affaires ${ }^{82}$ ou à des fusions ${ }^{83}$ et, donc, que c'est peut être là une condition infrastructurelle de la réalisation de la convergence au Canada. On sait également que l'adressabilité va, malgré la hausse du tarif de base qu'elle implique ${ }^{84}$, connaître un certain succès, ou mieux un succès certain, parce que: 1) les consommateurs à qui on la "propose" ont acquis l'habitude de choisir dans une programmation diversifiée ${ }^{85}$; 2) la substitution massive de la câblodistribution par d'autres produits n'est pas probable du fait de cet habitus et aussi du fait que ces produits concurrents sont également complémentaires et qu'ils peuvent être intégrés directement ou indirectement par la câblodistribution ${ }^{86} ; 3$ ) que la nouvelle technologie n'oblige pas le consommateur à passer par une rupture, mais situe la nouvelle pratique dans une continuité avec la pratique de choix et la logique de club qui dominent la production-consommation télévisuelle au Canada ${ }^{87}$; 4) que tout cela rend "l'élasticité" de la liberté d'adhérer et de consommer plutôt faible.

Sachant ces choses, on sait aussi que ce "succès" probable relève d'une construction, que nous avons appelée "traduction du projet d'informatisation sociale", dans laquelle, toutefois, les acteurs ne sont pas en position symétrique et en alignement "dialogique", comme le voudrait l'explication du processus d'innovation avancée par Callon et Latour. Finalement, tout cela fait que nous savons aussi que, malgré le succès probable de la généralisation envisagée par

82 Vidéotron et Bell Canada se sont associés pour exploiter en Angleterre un territoire. Ils y offrent un ensemble intégré de services et produits relevant à la fois des télécommunications et de la câblodistribution.

83 Comme en témoignent les récentes méga-fusions entre de grandes entreprises de câblodistribution et de télécommunications aux États-Unis. AFP, "La fusion Bell Atlantic-TCI consacre l'avènement de la télévsion interactive", Le Devoir, 14 novembre 1993, p. B-5.

84 G. Tremblay, J.-G. Lacroix, M. Ménard et M. Saint-Laurent, "Les abonnés du câble devront dépenser en moyenne deux fois plus", article cité.

85 Environ $70 \%$ des foyers québécois sont abonnés au câble et s'adonnent activement au "pitonnage".

86 J.-G. Lacroix, G. Tremblay, M. Ménard et M. Saint-Laurent, Télévision 2001: câblodistributeurs et étoiles de la mort, op. cit.

87 Voir à ce propos, G. Tremblay et J.-G. Lacroix, Télévision. Deuxième dynastie, op. cit. 
Vidéotron, ce ne sera pas l'entièreté des foyers qui adopteront la nouvelle technologie ${ }^{88}$, ce qui questionne la notion usuelle de généralisation. Le cas français est très significatif à cet égard.

L'implantation générale de Télétel et du minitel a connu un succès relatif ${ }^{89}$, comme en témoignent la multiplication par cinq du nombre de minitels installés (ils sont passés de 1,3 à 6,3 millions), le nombre de services, qui a augmenté de 10,5 fois, passant de 1900 à 20000 , le nombre d'appels qui a été multiplié par plus de dix (10,8), passant de 164 millions à près de 2 milliards $(1,775)$, et le nombre d'heures d'utilisation qui est passé de 14 à 110 millions ${ }^{90}$.

Cette implantation générale s'est déroulée, comme les périodes précédentes, sous le sceau du volontarisme et a été accompagnée d'un intense discours prescriptif dirigé à la fois vers le grand public, les professionnels et les fournisseurs de services. Afin de saisir la stratégie de l'acteur promoteur durant cette période et d'identifier les étapes de cette généralisation, nous avons effectué une analyse de contenu de la Lettre de Télétel ${ }^{91}$, qui fut et demeure un véhicule important du discours prescriptif de France Télécom.

Durant la première étape, de 1984 à 1987, France Télécom procède à l'extension du réseau télématique et à l'ouverture progressive du service de l'annuaire électronique à l'ensemble du territoire ${ }^{92}$. De plus, on rend le système de kiosque téléphonique, jusqu'alors réservé aux entreprises de presse et de communication, accessible à tout consommateur parisien. Et, on met en place l'infrastructure technique et administrative nécessaire pour diversifier et élargir, autour du service de l'annuaire électronique, la gamme des produits et services. Pendant ce temps, les usages commencent aussi à se diversifier et les applications utiles gagnent en popularité et commencent à se substituer aux applications ludiques (jeux et messageries) comme élément moteur du développement du marché. Finalement, en 1987, le trafic grand public semble plafonner alors qu'on enregistre une forte croissance des utilisations professionnelles, qui représentent alors près du tiers des utilisations.

88 Selon les estimations mêmes des câblodistributeurs, exposées lors des audiences du CRTC en mars 1993, environ $85 \%$ des foyers abonnés à la câblodistribution, c'est-àdire $70 \%$ des foyers québécois, accepteront la "proposition" des câblodistributeurs, ce qui représente environ $60 \%$ du total actuel des foyers québécois abonnés.

89 Un succès si on compare cette implantation à d'autres tentatives d'implantation de NTIC, par exemple Antiope et Alex, mais un succès relatif quand on tient compte du fait, qu'à ce moment, il y avait en France vingt millions de téléphones en service et qu'on a offert le terminal graduitement.

90 L'événement du jeudi, 11 au 17 mars 1993, p. 37.

91 L. Santerre, P. Mœglin et J.-G. Lacroix, Le discours prescriptif de France Télécom et la production des usages sociaux de la télématique, op. cit.

92 Rappelons que Vidéotron fait face à la même contrainte et que, à l'instar de ce que fit la DGT, il n'ouvre que progressivement les territoires qu'il dessert à sa formule télématique. 
Durant la deuxième étape, 1987-1988, on assiste à la montée des services professionnels. On offre des appareils plus performants (minitel bistandard: mode Télétel et ASCII), on lance un lecteur de cartes à mémoire (LECAM), on crée un kiosque professionnel multitarif et on tente d'internationaliser Télétel. C'est aussi à ce moment que la DGT, devenue France Télécom (1986), se repositionne de façon majeure dans l'économie en tant qu'acteur promoteur clé, ce qui fera que la forme de la mise en place de l'offre sera de plus en plus marchande, plutôt qu'articulée par la logique de service public ${ }^{93}$.

La troisième étape, de 1988 à 1991, se caractérise par une série de progrès techniques et d'améliorations concernant principalement les services professionnels. Parmi ces progrès mentionnons la création d'un kiosque d'informations professionnelles et l'instauration de paliers supplémentaires à très haut niveau de tarification; l'ouverture de l'annuaire des usagers du télex; la mise en place du télétex et de la télécopie SCRIP; le lancement d'une gamme d'un minitels à haute performance; l'implantation de l'acheminement et du reroutage en langage naturel et du télépaiement; la diffusion du minitel portatif. Ces progrès ont donné à la technologie proposée un caractère de plus en plus concret, appliqué et utile dans une diversité de milieux. Ils l'ont donc fait apparaître comme une technologie arrivée à maturité, représentation sur laquelle les différents promoteurs (le médiateur France Télécom autant que les fournisseurs services) vont s'appuyer pour affirmer qu'elle est incontournable, inéluctable, afin d'approfondir et élargir le marché, donc généraliser davantage.

Dans la quatrième étape, depuis 1991, un ensemble d'ajustements rend la nouvelle technologie encore plus diversifiée et plus largement accessible. On a ainsi mis l'accent sur la convivialité du système pour l'usager en augmentant les possibilités d'utilisation en langage naturel, développé le Télétel Vitesse Rapide qui permet un traitement plus performant de l'image; mis au point un minitel Photo, créé les services vocaux Audiotel. Et la perspective du traitement numérique intégré de l'image et du son se profile comme une réalité de plus en plus immédiate compte tenu que les services téléinformatiques supportés par l'ensemble des accès Télétel ont de plus en plus autorisé l'intégration des services. Tous ces développements et améliorations se sont inscrits dans une perspective de plus en plus marchande ${ }^{94}$. Le reroutage est devenu payant. De la publicité a été introduite dans les pages du Minitel Guide des services (MGS) et France Télécom

93 Parallèlement, la conversion de l'acteur "PTT" en "France Télécom" visait à poser cet organisme en interlocuteur obligé de tous les acteurs économiques intervenant dans le secteur en France ainsi que par rapport à l'étranger.

94 Cette perspective existait déjà depuis un certain temps. Ainsi, en ce qui concerne la mise en circulation du terminal minitel, France télécom a tenté à plusieurs occasions de changer sa stratégie de départ consistant à le distribuer gratuitement. Finalement, devant les protestations, le promoteur a adopté comme tactique de dire que "le minitel gratuit" n'était plus disponible afin de contraindre les usagers à choisir d'autres minitels, ceux-là vendus ou loués. 
a créé Marketis pour l'utilisation, à des fins de marketing direct, des données de l'annuaire électronique. Finalement, on a commencé à vendre certains minitels et le LECAM.

La liste des innovations, des progrès, des ajouts, des ajustements techniques et techno-économiques effectués durant cette période, encore ouverte, montre que la technologie Télétel a pénétré une diversité élargie de pratiques et de fonctions sociales tout en s'unifiant et en s'articulant en un système technique capable de réorganiser l'interaction entre plusieurs secteurs d'activités. L'évolution quantitative et qualitative de la fonction d'éditeur télématique est un bon indicateur de cette progression. En effet, aux premiers moments de Vélizy, en 1981, on dénombrait environ 80 éditeurs de services, ils sont aujourd'hui plus de 5200 qui se partagent plus de 20000 codes d'accès sur le marché Télétel; de sorte qu'il n'y a maintenant que très peu de secteurs, surtout dans les différents domaines du tertiaire, qui ne se soient pas faits de place dans ou qui n'ont pas été attirés par l'univers Télétel ${ }^{95}$. Même si dans bien des secteurs, le service vidéotex demeure secondaire, on doit retenir que la technologie Télétel s'est inscrite dans la quotidienneté du travail d'une diversité élargie de secteurs d'activité et dans une grande partie des foyers français. On peut donc parler d'une certaine banalisation de la technologie avancée par France Télécom et ses partenaires. C'est ce qui nous amène à penser que les objets techniques, les contenus qui les accompagnent et les pratiques qui les entourent commencent à atteindre le stade de la norme sociale.

Plusieurs indicateurs nous invitent à orienter notre réflexion dans cette direction. Nous en retiendrons deux. Premièrement, de l'avis même du promoteur, il ne resterait plus pour le minitel de base (minitel 1) qu'un espace potentiel de deux à trois millions d'unités ${ }^{96}$. Puisqu'il y a déjà près de 6,5 millions de minitels installés, cela implique que les deux tiers du marché potentiel auraient été occupés. On serait donc en train d'atteindre la capacité maximale d'absorption de la société française, capacité, notons-le, qui ne recouvre pas l'entièreté de la population, comme ce sera probablement le cas pour Vidéoway, ainsi que nous l'avons déjà fait remarquer. Le deuxième indicateur est corollaire au premier, mais nous semble davantage décisif. De l'avis de gens du milieu, en même temps que la demande est en voie de se stabiliser, les producteurs de services sont de plus en plus confrontés à des attentes de la part de consommateurs de vidéotex de plus en plus avertis ${ }^{97}$. Cela implique que l'offre commence à faire face à un ensemble de consommateurs qui ont pris l'habitude de la "consommer" et qui ont intégré les savoirs requis pour une utilisation courante. Il s'agit donc de consommateurs qui ont été ou qui se sont socialisés à cette offre. C'est par cette socialisation - qui cristallise les

95 P. Brouillard, P. Pajon, P. Mœglin, G. Tremblay et J.-G. Lacroix, Les éditeurs du vidéotex français sur le marché des services grand public, Montréal, GRICIS, 1993.

96 Vidéotex, La newsletter des professionnels du vidéotex, no 227, 6 mai 1992, p. 1.

97 Entrevue avec un responsable de la télématique d'un groupe de presse. P. Brouillard et al., Les éditeurs du vidéotex français sur le marché des services grand public, op. cit., p. 51 et 52 . 
utilisations en habitudes, puis en besoins et attentes et finalement, éventuellement, en exigences d'amélioration - que l'offre opère un retour sur ellemême par le biais des consommateurs socialisés, ce qui indique que le processus atteint alors le niveau de la régulation sociale ${ }^{98}$. On peut considérer qu'à ce moment le processus fait un saut qualitatif par l'enclenchement d'un interaction dialectique entre les composantes du marché, entre la production-distribution et la consommation. Ce qui permet aux partisans et idéologues de l'offre-demande, comme rhétorique explicative et justificatrice des rapports entre les offreurs et les consommateurs, d'avoir le sentiment d'être "dans le vrai" et de pouvoir, en toute "conscience", ne pas voir le processus socio-historique de mise en place de l'offre des NTIC et de production des usages... sociaux.

Mais pour comprendre comment cette socialisation permet à l'offre d'opérer un retour sur elle-même et apparaît sur le marché et dans l'espace public comme une demande engendrée par des besoins et comment tout cela constitue la "terre promise" de la convergence, il faut revenir un peu en arrière. Il faut se souvenir que le processus commence par la production d'un discours social véhiculant un projet de société, lequel ne peut apparaître comme socialement pertinent et légitime que s'il se présente et est perçu comme le moyen de réaliser les grandes valeurs structurant la conscience dans les sociétés capitalistes contemporaines, c'est-à-dire, entre autres, la liberté, l'individualité, la démocratie... et le progrès, qu'on inscrit dans un rapport d'équivalence avec bonheur et bien-être. Il faut également se souvenir que ce projet sera traduit en réalités concrètes -- d'abord expérimentales, puis prototypes, par la suite en objets soumis à l'épreuve de l'adoption sociale et enfin en objets offerts à tout le monde à la masse des consommateurs - qui vont toujours être différentes et toujours renfermer une capacité-utilité différente de celle annoncée par le projet parce que ce dernier est traduit par des acteurs inspirés par des réseaux de déterminations objectives, particulièrement sur les plans économique et technique. Il y a donc toujours un écart entre le projet et les réalisations techno-économiques offertes, ce que nous avons nommé la mise en place de l'offre. De plus, parce que le discours prescriptif - chargé tout au long du processus de formation des usages sociaux, d'atténuer autant que possible ce décalage de même que celui que l'antériorité de l'offre tend toujours à induire entre utilisations prescrites et utilisations effectives - ne peut avoir de prégnance qu'en faisant appel au projet et aux valeurs qui le légitiment, ce même discours ne peut à terme que faire naître des attentes que ne peuvent satisfaire les objets et les pratiques offertes, surtout quand la patine de la quotidienneté et de la récurrence a effacé le lustre de la nouveauté et l'a remplacé par la banalisation. Il faut aussi tenir compte du fait que la pénétration d'une nouvelle technologie, d'un nouveau média, dans un champ de pratique sociale spécifique (par exemple l'éducation) ne se fait qu'à travers des ajustements qui relèvent de l'interaction entre la forme dans

98 À propos de l'effet de rétroaction de la consommation des NTIC sur la sphère de la production, voir L. Santerre, La formation des usages sociaux de la micro-informatique domestique, thèse de doctorat, Montréal, département de sociologie, Université du Québec à Montréal, 1993. 
laquelle l'offre se présente aux différents champs de pratiques, les conditions d'action dans ces derniers, les besoins qui les caractérisent (le besoin d'éducation) et l'utilité (avantage) que les acteurs dans ce champ finissent par attribuer à la proposition technologique ${ }^{99}$. Finalement, il faut également prendre en compte le fait de la domination grandissante, voire hégémonique, de l'image ${ }^{100}$ comme mode d'expression, de transmission et d'interprétation (d'appropriation, il s'agit donc d'un habitus, d'un critère d'intelligibilité) de l'information.

L'interaction de tous ces facteurs condamne la technologie Télétel-minitel à évoluer, ce qui fait que la convergence technologique est en France aussi à l'ordre du jour

"Au départ nous avions le téléphone, après on a eu l'informatique, puis on s'est dit: "Bon, on pourrait intégrer les deux (...) on va voir ce que ça donne". (...) L'informatique reste toujours un peu sinistre dans l'esprit des gens. Mais, l'audiovisuel devient un troisième partenaire qui va nous apporter un peu de gaieté, un peu de rêve dans tout ça. Je pense que ça devrait faire un très beau mélange ${ }^{101}$."

Ce avec quoi Vidéotron est tout à fait d'accord, comme le montre sa publicité qualifiant son système télématique audiovisuel " $d$ 'univers merveilleux ${ }^{102}$ ".

\section{Conclusion}

Au terme de cet article, faisons un bref retour sur certaines questions soulevées par notre analyse.

Un de nos objectifs était d'identifier les périodes constitutives du processus d'informatisation sociale. Les études que nous avons réalisées nous ont amenés à repérer six périodes, la dernière, la généralisation, étant encore ouverte. Nous

99 À ce propos, voir P. Mœglin, "La notion de besoins éducatifs: remarques sur l'introduction des nouveaux médias dans l'éducation", Bulletin de l'IDATE, no 19, 1986, p. 89-99.

100 Ici, par image, nous désignons plus que ce qui est désigné habituellement par le mot image. Il s'agit de toutes les formes de représentation globalisante, intégrative, schématisante, autonomisante (séparant) et analogisante d'une partie, plus ou moins grande de la réalité à laquelle appartient ce qui est représenté. Il s'agit donc d'un mode extensif de fixation de la représentation. Ainsi, une partie de texte à l'écran est une image du texte; un transparent (acétate, au Québec) qui supporte un texte, un graphique, un tableau ou un dessin, est une image, etc.

101 Entrevue avec une responsable télématique d'une grande agence de voyage. P. Brouillard, P. Pajon, P. Mœglin, G. Tremblay et J.-G. Lacroix, Les éditeurs du vidéotex français sur le marché des services grand public, op. cit., p. 82.

102 P. Rigaud et J.-G. Lacroix, Vidéotron-Vidéoway. Discours promotionnels et prescriptions d'usages, op. cit. 
tenons toutefois à rappeler que notre périodisation n'obéit pas à un quelconque modèle théorique du processus d'implantation de l'innovation. Elle résulte plutôt d'une analyse, inspirée de l'économie-politique, qui cherchait à repérer les transformations de l'interaction entre les acteurs impliqués dans le processus et de la nature des actions structurantes qui infléchissent la trajectoire de réalisation du projet d'informatisation sociale à l'origine (période 1) du processus. Notre périodisation a donc la même limite que tout découpage empirique, c'est-à-dire qu'elle ne peut prétendre au statut de modèle, ce qui n'était d'ailleurs pas notre intention. Par contre, elle a le mérite de montrer que le procès d'informatisation sociale n'est ni homogène ni continu ni régulièrement progressif et qu'il ne peut s'expliquer par la seule force cinétique de la technologie. Au contraire, notre périodisation met bien en lumière que le processus est scandé par des moments où l'interaction des acteurs impliqués est relativement stable et homogène et des moments de transformation de l'interaction, de rupture, où la direction de la trajectoire et le rythme de réalisation, le nombre, la nature et le poids des acteurs impliqués dans l'action structurante changent.

De fait, il s'agit d'un procès social marqué par de véritables mutations de l'action, laquelle, d'étape en étape, change de nature et de terrain. Ainsi, au départ, l'action structurante est constituée du discours social sur le projet d'informatisation de la société, discours qui a comme rôle de préparer les esprits, de convaincre la collectivité de la nécessité et du caractère incontournable, inévitable, des nouvelles technologies d'information et de communication. Par la suite, la partie structurante de l'action, c'est-à-dire celle qui fait progresser le projet sur une trajectoire de réalisation, n'est plus le discours, que nous avons qualifié de politico-prospectif, mais l'amorce de la traduction du projet par la mise au point concrète d'une technologie, d'une norme technique (en l'occurrence, dans nos études, Télétel et Télidon). Cela ne veut toutefois pas dire que le discours ne joue plus de rôle actif, mais, qu'à ce moment du processus, l'action déterminante de son déroulement est autre. Puis, avec la fin de cette deuxième période, l'action qui devient structurante est encore différente; et, ainsi de suite tout au long de l'enchaînement des périodes.

Il en va de même pour les acteurs jouant un rôle structurant. Dans la première période, l'État lance le processus en animant et régulant les opérations d'établissement du diagnostic de crise, de recherche de solution, de cristallisation d'un projet collectif de réponse à la problématique de crise structurelle. Puis, avec la deuxième période, on change d'acteur au poste de commande, la recherchedéveloppement prend le relais. L'État est encore présent et actif, mais la progression sur la trajectoire de réalisation, de concrétisation du projet, est due à un autre acteur. Dans la période suivante: d'une part, l'acteur dont l'action est structurante dans la seconde période doit s'associer à des partenaires, ce qui entame le processus de socialisation de la mise en place de l'offre; et, d'autre part, on met socialement à l'essai l'objet technique mis au point et matérialisant la norme technique, elle-même mise au point dans la période précédente, ce qui fait entrer dans le processus le public en tant qu'acteur réellement actif. Puis, avec la fin de 
cette troisième période, l'acteur qui acquiert un poids structurant est encore différent; et, ainsi de suite tout au long de l'enchaînement des autres périodes.

Ainsi, dans les cas que nous avons étudiés, de période en période, les acteurs faisant avancer le processus et l'action structurante de son déroulement seront:

\begin{tabular}{|c|c|c|}
\hline Périodes & Le-les acteur(s) & L'action structurante \\
\hline 1 & $\begin{array}{l}\text { l'État (des experts, des idéologues } \\
\text { et du personnel politique) }\end{array}$ & le discours politico-prospectif \\
\hline 2 & $\begin{array}{l}\text { l'État et certaines entreprises } \\
\text { (la recherche-développement) }\end{array}$ & $\begin{array}{l}\text { la mise au point d'une techno- } \\
\text { logie (la norme technique) }\end{array}$ \\
\hline 3 & $\begin{array}{l}\text { des entreprises promotrices } \\
\text { (la recherche-développement) }\end{array}$ & la mise au point d'un prototype \\
\hline 4 & $\begin{array}{l}\text { l'entreprise promotrice princi- } \\
\text { pale (la recherche-développement } \\
\text { et la production) et des fournis- } \\
\text { seurs de services (partenaires) et } \\
\text { une partie restreinte du grand } \\
\text { public }\end{array}$ & $\begin{array}{l}\text { l'ajustement de l'offre et sa } \\
\text { diversification sur la base des } \\
\text { réactions du public }\end{array}$ \\
\hline 5 & $\begin{array}{l}\text { l'entreprise promotrice princi- } \\
\text { pale et des fournisseurs de } \\
\text { services (production et marke- } \\
\text { ting) et une partie élargie, mais } \\
\text { restreinte, du grand public }\end{array}$ & $\begin{array}{l}\text { le discours et les actions prescrip- } \\
\text { tives et la publicité et l'adoption } \\
\text { de la proposition par une } \\
\text { première masse de consomma- } \\
\text { teurs }\end{array}$ \\
\hline 6 & $\begin{array}{l}\text { l'entreprise promotrice principa- } \\
\text { le et les fournisseurs de services } \\
\text { (marketing) et les consommateurs } \\
\text { comme généralité }\end{array}$ & $\begin{array}{l}\text { le discours et les actions prescrip- } \\
\text { tives et la généralisation }\end{array}$ \\
\hline
\end{tabular}

En plus d'illustrer les changements de l'action sociale par laquelle se réalise le processus d'informatisation sociale, ce tableau synthèse laisse entrevoir la très grande complexité du processus qui est "habité", animé, traversé, par plusieurs processus. Ainsi, le discours politico-prospectif qui lance le processus 
d'informatisation sociale relève du procès de régulation et de gestion sociale. Par la suite, avec l'amorce de la traduction du projet s'enclenche un procès de sélection sociale des promoteurs et des propositions. S'ajoute dans le cursus de la mise au point de l'offre un procès de socialisation de l'offre avec l'intégration de partenaires. Le processus de construction d'une représentation sociale concrète va venir interagir avec les autres procès déjà en cours. Puis, le processus d'ajustement, d'affinement et de diversification de l'offre va aussi entrer en interaction avec la construction de la représentation. Finalement, le procès de formation des usages sociaux chapeaute cette complexité. Et, si l'on tient compte du fait que non seulement ces différents processus mettent à contribution des acteurs souvent très différents, mais qu'ils opèrent selon des temporalités différentes, on comprend que toute cette dynamique est encore plus complexe qu'elle n'apparaît à première vue, ce qui renvoie au problème du repérage des facteurs déterminant les actions structurantes du processus.

Nous avons plus haut parlé de trajectoire, de traduction du projet, de concrétisation, de cristallisation, etc., ce sont là tous des termes qui peuvent donner l'impression que nous concevons le déroulement du processus comme une lignée de réalisations déterminées à partir du projet et que notre approche est déterministe. Au contraire, notre analyse montre bien qu'il s'agit d'un procès de structuration, de détermination, graduelle. Cette structuration est à la fois déterminée par le déjà structuré (particulièrement des conditions infrastructurelles, des habitudes de consommation et des rapports sociaux) et non déterminée parce que ce qui est structuré n'est pas homogène mais complexe et diversifié et que cela implique qu'il y a d'autant plus de possibilités de réponse et de sujets acteurs de réponse, de propositions, que le tissus social est diversifié et complexe, comme c'est le cas des sociétés où la problématique de l'informatisation sociale se pose avec acuité. De plus, en tenant compte du fait que le déjà structuré ne détermine que par l'intermédiaire de certaines subjectivités, autant au niveau du politique, qu'à celui des acteurs industriels (les stratégies d'entreprise), qu'à celui des consommateurs (les goûts et préférences), on s'aperçoit que le processus ne peut progresser qu'à travers un réseaux de différences et de variations. Celles-ci ne doivent toutefois pas être conçues comme individuelles parce que les sujets sont toujours insérés dans un réseau social de détermination - constitué de mécanismes sociaux, tels que la socialisation, et des conditions infrastructurelles - qui façonne leur subjectivité et fait que ces différences et variations sont toujours exprimées par des ensembles plus ou moins grands d'individus, donc qu'elles sont toujours l'expression d'une certaine collectivité.

Notre analyse nous place ainsi en profonde divergence avec les thèses diffusionnistes - même si dans les versions "amérioaméliorées", par exemple la deuxième approche de Rogers ${ }^{103}$, on tient compte des ajustements dus aux

103 Voir à cet effet E. M. Rogers, Diffusion of Innovations (3e édition), New York, The Free Press, 1983; et du même auteur, Communication technology. The New Media in Society, New York, The Free Press, 1986. 
réactions des individus - ou encore associationnistes ${ }^{104}$ qui sont édifiées sur le postulat que l'individu à qui l'offre soumet un objet techno-économique est libre d'accepter ou de refuser cette proposition. Au mieux, ces thèses tiennent compte des habitudes, des conditions socioéconomiques variées de réception-adoption ou encore de la socialisation comme facteurs de détermination du "choix" des individus. Cependant, elles oublient que ces facteurs peuvent et sont de fait transformés par l'offre, comme l'a montré notre analyse. Ainsi, ce sont ces différents mécanismes sociaux, "travaillés" par l'offre, particulièrement la socialisation, - on comprend ainsi toute l'importance du "dossier" de l'informatisation scolaire et de l'informatique domestique - qui expliquent que "l'élasticité" de la liberté des individus d'adopter ou non, est, à terme, tôt ou tard, plutôt faible. Le postulat servant de fondation idéologique à ces thèses permet toutefois de "donner" aux individus-consommateurs le statut d'acteur égal à l'offre. Notons que la thèse traductionniste fonctionne sur la même base, avec la même conséquence idéologique. En effet, ces deux familles de thèses, en oubliant l'effet contraignant du processus, en taisant l'efficace du contrôle social dans le processus d'implantation et de généralisation des NTIC, cachent que ce procès social, particulièrement dans les moments de rupture et de réalignement de la trajectoire de réalisation, passe par des rapports de force, de pouvoir, dans lesquels les sujets de l'interaction ont un poids inégal, comme notre analyse l'a montré et contrairement à ce que soutiennent Callon et Latour en affirmant qu'ils sont en alignement symétrique ${ }^{105}$.

Finalement, deux dernières remarques: la première sur la notion de généralisation, la seconde sur celle de convergence.

Notre analyse indique que l'adoption des propositions technologiques que nous avons étudiées n'est pas (Télétel) et ne sera probablement pas (Vidéoway) le fait de l'entièreté de la population. Or, dans notre problématique, nous avons défini les usages sociaux comme des utilisations récurrentes organiquement insérées dans la quotidienneté de la généralité des citoyens. Le critère de l'insertion dans la vie courante de la collectivité devrait nous amener à conclure qu'il n'y a pas de généralisation et pas d'usages sociaux parce que ce n'est pas la totalité de la population qui adopte et utilise de façon récurrente la proposition technoéconomique. Au contraire, en nous appuyant sur le fait que l'offre intégrée Télétel commençait à devoir affronter des attentes d'utilisateurs avertis, nous avons conclu qu'il y avait une certaine banalisation de la technologie dans plusieurs milieux, au niveau du travail comme à celui de la vie privée, et que cela témoignait d'une socialisation suffisante des individus à la technologie pour faire atteindre à sa présence dans la société et aux utilisations dont elle est l'objet le niveau de la

104 G. A. Barnett, An Associational Model for the Diffusion of Complex Innovations, présentation faite à l'Association internationale de communication, Chicago (II), 1978.

105 M. Callon et B. Latour, "Les paradoxes de la modernité. Comment concevoir les innovations?", op.cit., p. 9. 
norme sociale. Notre analyse nous conduit donc à conclure qu'il y a généralisation parce que, par le biais de la socialisation et du contrôle social, c'est la majorité, sinon l'ensemble des individus constituant la collectivité, ici française, qui est touchée, familiarisée, amenée à utiliser, personnellement ou par personnes proches (foyer) ou interposées (organisation du travail), directement ou indirectement, les ou des objets concrétisant l'univers Télétel.

Ce qui nous amène à poser la question suivante: s'il y a généralisation sans que la totalité de la population adopte et utilise directement la totalité de l'univers Télétel - et nous pensons que le raisonnement vaut également pour Vidéoway qu'est-ce qui est généralisé et qu'est-ce qui est érigé en norme sociale?

En tenant compte de la diversité des propositions (diversité et concurrence des filières, des produits et des services) et de la diversité intégrée par la proposition principale et motrice du développement du procès d'informatisation (découlant du procès de sélection sociale des propositions et des acteurs), on s'aperçoit que cette familiarisation-appropriation - qu'on pourrait qualifier de degré minimal de socialisation aux NTIC - peut se faire par le biais d'une diversité-multiplicité d'objets techno-économiques et de pratiques qui concrétisent l'informatisation sociale. Toutefois, cette familiarisation-appropriation minimale aussi partielle soit-elle permet aux citoyens de faire face aux autres objets de l'univers de l'informatisation et de les utiliser directement ou indirectement de façon au moins minimale. Elle implique plus ou moins directement les individus composant une collectivité dans le processus d'informatisation, ce qui donne au projet de société informatisée une matérialité certaine et une apparence de réalité sociale vécue par la généralité des individus. Cette familiarisation se révèle ainsi le levier qui fait basculer la collectivité en général dans l'univers de l'informatisation sociale parce que sans faire de l'ensemble des individus des acteurs actifs du processus et des demandeurs d'amélioration, elle les place dans une position de consensus minimal, d'acceptation passive ou d'utilisation minimale. C'est à ce moment que le procès d'informatisation sociale est banalisé et que les objets et utilisations qui le concrétisent sont cristallisés en norme sociale, même si le mouvement d'ajustements, d'innovations, d'ajouts, de cumul, d'articulation en système se poursuit.

S'ajoute une appropriation approfondie mais variable selon les acteurs, les occupations professionnelles, les revenus, etc. Cet approfondissement de la maîtrise de certains produits et services informatiques exerce une pression à la hausse sur le niveau minimal d'appropriation d'autant plus forte qu'elle se fait dans une grande diversité de champs de pratique. À terme, elle entraîne un approfondissement de l'appropriation-familiarisation minimale "normée"106 et pose tout aussi objectivement la convergence que l'état de la technique (la

106 Un peu comme le développement des connaissances dans certains milieux et le développement et la généralisation de nouvelles techniques d'expression de la pensée (l'écriture, l'imprimerie, etc.) élèvent le niveau de l'alphabétisation relative. 
numérisation), les intérêts économiques ou encore certaines volontés parce qu'elle "prépare" les individus à cheminer dans les ramifications et la diversité de l'univers de l'informatisation.

Notre démarche analytique nous ramène donc à notre point de départ, la convergence, et nous conduit à nous demander ce qui est vraiment en convergence, si convergence il y a.

Nous avons déjà souligné l'ambiguïté du terme convergence ${ }^{107}$. Notre analyse montre qu'il y a des d'objets et des ensembles d'objets qui se rapprochent et qui tendent à s'artïculer en système intégré mais aussi de plus en plus diversifié, qu'il y a des ponts qui sont jetés par la numérisation, et qu'à cette diversité en voie d'articulation correspond une diversité de pratiques entre lesquelles la familiarisation-appropriation minimale jette également des ponts. Tout cela, en fait, indique que convergence veut dire: diversification, ramification, articulation, généralisation. Le terme convergence indiquerait donc que le procès d'informatisation sociale arrive à un moment de concrétisation en tant que réponse collective unifiée à la problématique posée au départ par la cristallisation du projet de société informatisée. Nos données et notre analyse suggèrent en effet que le procès serait sur le point d'atteindre un état de maturité, qu'il serait en train d'entrer dans une phase de généralisation-approfondissement qui devrait durer tant et aussi longtemps - ici, nous faisons référence à un éventuel cycle long de croissance que la société ne se sera pas posée une autre question structurante.

“(...) tout processus d'équilibration cesse, tôt ou tard, de constituer la réponse optima au besoin de trouver un équilibre significatif entre le sujet collectif et son monde ambiant. Apparaîtront alors, à l'intérieur même du processus initial, des processus de structuration orientés vers un nouvel équilibre différent de l'ancien et mieux adapté à la praxis actuelle du groupe ${ }^{108}$."

Jean-Guy LACROIX, Département de sociologie Gaëtan TREMBLAY, Département de communications, Université du Québec à Montréal

Gilles PRONOVOST, Département des sciences du loisir Université du Québec à Trois-Rivières

107 J.-G. Lacroix, B. Miège, P. Mœglin, P. Pajon et G. Tremblay, "La convergence des télécommunications et de l'audiovisuel. Un renouvellement de perspective s'impose", article cité.

108 L. Goldmann, Epistémologie de la sociologie, J. Piaget (dir.), Logique et connaissance scientifique, Paris, Gallimard, La Pléiade, 1967, p. 1000. 


\section{Résumé}

Après avoir situé l'interaction entre la mise en place de l'offre des nouvelles technologies d'information et de communication (NTIC) et la formation des usages sociaux dans le contexte de la convergence entre télécommunicationscâblodistribution, les auteurs se donnent comme objectif d'identifier les étapes périodisant le processus d'implantation et de généralisation de la télématique, de repérer les acteurs ayant un poids structurant dans le déroulement du processus et de saisir l'action déterminante de son évolution dans chacune des périodes. Pour y parvenir, les auteurs utilisent les résultats de deux enquêtes: l'une ayant porté sur minitel; l'autre sur le cas de Vidéoway. Six périodes sont décrites et analysées: 1) l'élaboration du projet social informatique où l'État joue le rôle central; 2) l'établissement d'une norme technique et la prise en charge par des acteurs industriels de la réalisation concrète du projet; 3 ) la mise au point d'un prototype et sa mise à l'essai sociale qui permet d'affiner la proposition technique; 4) l'amorce de la socialisation de la nouvelle technologie par des expérimentations grandeur nature, grand public, ce qui marque l'entrée en scène des usagers en tant qu'acteurs réellement actifs dans le déroulement du processus; 5) la première phase de commercialisation, durant laquelle se poursuit la mise au point de l'offre, particulièrement au niveau des contenus, et par laquelle l'offre se donne une masse critique de consommateurs, ce qui lui permettra de légitimer ses efforts de généralisation; 6) finalement, la généralisation qui se réalise sans toutefois que l'entièreté de la population soit touchée et qui se confond avec un mouvement de diversification et de pénétration dans un ensemble élargi de pratiques sociales. En conclusion, les auteurs reviennent sur les conditions de généralisation de l'informatisation et sur le rôle de la convergence comme moment décisif du processus d'informatisation.

mots-clés: Nouvelles technologies, informatisation sociale, télématique, télécommunications, câblodistribution, convergence, usages sociaux, structure industrielle, stratégie d'acteurs, État, socialisation.

\section{Summary}

After having situated the interaction between the implementation of new information and communication technology (NICT) supply and the creation of social uses within the context of the convergence of telecommunications and cable distribution, the authors have as their objective the identification of the stages periodizing the implementation and generalization of telematics, the identification of actors having a structuring role throughout this process, and the understanding of the action determining its evolution during each stage. To this end, the authors draw from the results of two studies: one of Minitel, the other of Videoway. Six periods are described and analyzed: 1) the elaboration of a social project of computerization in which the state plays the central role: 2) the establisment of a technical norm and the taking charge of the concrete realization of the project by 
industrial actors; 3) the creation and testing of a prototype, which permits the refinement of the technological proposition; 4) the initiation of the socialization of the new technology by means of large scale experiments implicating the public at large, which mark the entrance of users as actors truly active in the ongoing process; 5) the first phase of commercialization during which the suppli is refined, particularly at the level of content, and in which the supply achieves a critical mass of consumers, thereby justifying its generalization efforts; 6) finally, the generalization is accomplished without, however, affecting the entire population, and is merged with a movement of diversification and penetration into a larger set of social practices. In conclusion, the authors underline the conditions of the generalization of computerization and the role of convergence as the decisive moment in the process of computerization.

Key words: new technologies, social computerization, telematics, telecommunications, cable distribution, convergence, social uses, industrial structure, actor's strategies, State, socialization.

\section{Resumen}

Luego de haber situado la interacción entre la instauración de la oferta de las nuevas tecnologías de información y de comunicación (NTIC) y la formación de los usos sociales en el contexto de convergencia entre telecomunicaciones y cabledistribución, los autores se dan como objetivo identificar las etapas, periodizando los procesos de implantación y de generalización de la telemática; localizar los actores que tienen un peso estructurante en el desarrollo del proceso y comprender la acción determinante de su evolución en cada uno de los períodos. Para lograrlo, los autores utilizan los resultados de dos encuestas: una sobre minitel, la otra sobre el caso de Videoway. Seis períodos son descriptos y analizados: 1) la elaboración del proyecto social informático en el que el Estado juega un rol central; 2) el establecimiento de una norma técnica y la toma a cargo por actores industriales de la realización concreta del proyecto; 3) la puesta a punto de un prototipo y su ensayo social que permite afinar la proposición técnica; 4) el inicio de la socialización de la nueva tecnología a través de experimentaciones a escala natural (el público en general), lo que marca la entrada en escena de los usuarios en tanto actores realmente activos en el desarrollo del proceso; 5) la primera fase de comercialización, en la cual se prosigue la puesta a punto de la oferta, sobre todo a nivel del contenido y en la que la oferta adquiere una masa crítica de consumidores, lo que le permitirá legitimar sus esfuerzos de generalización; 6) finalmente, la generalización, que se realiza sin que por ello la población completa sea afectada y que se confunde con un movimiento de diversificación y de penetración en un conjunto ampliado de prácticas sociales. En conclusión, los autores retoman la discusión sobre las condiciones de generalización de la información y sobre el rol de la convergencia como momento decisivo del proceso de información. 
122 L'innovation technologique

Palabras claves: Nuevas tecnologías, información social, telecomunicaciones, cabledistribución, convergencias, usos sociales, estructura industrial, estrategia de actores, Estado, socialización.- 